\title{
«SAGESSE INCRÉÉE» ET «SAGESSE PARTICIPÉE» SELON MAÎTRE ECKHART
}

Fecha de recepción: 26 de julio de 2021

Fecha de aceptación: 23 de septiembre de 2021

RESUME: Nous proposons ici une première étude de la notion de sagesse selon Maître Eckhart, à partir de ses écrits latins, notamment son Commentaire du Livre de la Sagesse (In Sap. n.121, 201-206, 243 et 272-274), Les sermons et leçons sur l'Ecclésiastique (In Eccli.), et les sermons latins Sermo die b. Augustini Parisius habitus et le Sermo LV, ainsi que les Sermons allemands 95a, 95b et 112. Dieu, sa nature, ainsi que le Fils sont désignés par Eckhart comme Sagesse divine (sapientia increata), qui est aussi la source de toute sagesse humaine (sapientia participata). Au-delà de la sagesse terrestre ou naturelle, il y a une autre sagesse qui vient de la présence de Dieu, «une sorte de savoir savoureux», l'avant-goût de la douceur divine, qui n'est autre que la naissance de Dieu, au-delà des facultés humaines, au fond de l'âme.

MOTS CLÉS: Maître Eckhart; sagesse; Dieu; vertus; naissance éternelle.

\section{"Uncreated Wisdom» and "Participated Wisdom» according to Meister Eckhart}

ABSTRACT: We propose here a first study of the notion of wisdom according to Meister Eckhart, based on his Latin writings, notably his Commentary on the Book of Wisdom (In Sap. n.121, 201-206, 243 and 272-274), the Sermons and Lessons on Ecclesiasticus (In Eccli.), and the Latin Sermons: Sermo die b. Augustini Parisius

Universidad Pontificia Comillas: sbara@comillas.edu;

ORCID: https://orcid.org/0000-0002-6623-629X 
habitus and Sermo LV, as well as the German Sermons 95a, 95b and 112. God, his nature, and the Son are referred to by Eckhart as divine wisdom (sapientia increata), which is also the source of all human wisdom (sapientia participata). Beyond earthly or natural wisdom, there is another wisdom that comes from the presence of God, "a kind of tasty knowledge", the foretaste of the divine sweetness, which is none other than the birth of God, beyond the human faculties, in the ground of the soul.

KEY WORDS: Meister Eckhart; wisdom; God; virtues; eternal birth.

La $^{1}$ prière de Maître Eckhart (ca. 1260-1329), recopiée par un bénédictin de Melk au XV ème siècle, témoigne la grande estime du Dominicain thuringien pour la sagesse, car il la mentionne deux fois en huit lignes:

"Ô richesse très élevée de la nature divine,

montre-moi ton chemin, celui que tu as acquis dans ta sagesse,

et ouvre-moi le si précieux trésor auquel tu m'as appelé:

comprendre avec intelligence,

au-dessus de toute créature,

aimer avec les anges et jouir,

hériter avec ton Fils unique, notre Seigneur Jésus-Christ, te recevoir selon ta Sagesse éternelle»².

De plus, il soutient que «la sagesse dans l'âme transporte et transforme l'âme en Dieu ${ }^{3}$. Mais qu'est-elle pour lui? Se réfère-t-il à la Sagesse

1 Recherches effectuées dans le cadre du projet Teaching and Preaching with $\mathrm{Pa}$ tristic auctoritates. Meister Eckhart in France and Germany, financé par le programme en sciences humaines et sociales ANR-17-FRAL-0002, à l'Université de Lorraine.

2 Notre traduction, à partir de l'original en moyen haut allemand offert par Wolfgang Wackernagel. Poésies mystiques et prière de Maître Eckhart. Genève: Ad Solem, 1998, 114, qui reprend la transcription du manuscrit de Melk, publiée pour la première fois par F. Löser.

3 Eckhart. Sermo 55, LW 4 n.538, 452,5 (traduction française d'Éric Mangin [abrégé: EM], 433) «sapientia in anima transplantat et transformat animam in deum». Nous suivons l'édition critique des œuvres d'Eckhart. Die deutschen und die lateinischen Werke. 10 vol., Die deutschen Werke, édition de Josef Quint, et Georg Steer [abrégé: DW]; Die lateinischen Werke, édition de Ernst Benz, et al. [abrégé: LW]. Stuttgart: Kohlhammer, 1936-2015. Entre parenthèse, nous signalerons leurs traductions françaises, si elles existent: Commentaire du Livre de la Sagesse, traduction de Jean-Claude Lagarrigue, Jean Devriendt. Paris: Les Belles Lettres, 2015 [abrégé: JCL]; Sermons et leçons sur l'Ecclésiastique, traduction de Fernand Brunner. Genève: Ad Solem, 2002 [abrégé: B]; Commentaire de la Genèse, précédé des Prologues. L'ouvre latine de Maître Eckhart 1, traduction de Fernand Brunner et al. Paris: Cerf, 1984 
divine ou à la sagesse humaine? Et comment entend-il le rapport entre les deux, la sagesse incréée et la sagesse participée?

Malgré son grand intérêt, il s'agit d'un aspect de la pensée eckhartienne encore peu étudiét . Cependant, aborder la notion de sagesse chez le Maître thuringien permet de mettre l'accent sur des textes moins connus, en particulier son œuvre latine, et offre une nouvelle porte d'entrée à la pensée eckhartienne, qui permet de l'ancrer dans la tradition biblique, patristique et philosophique, mais aussi de montrer son originalité.

La réflexion d'Eckhart sur la sagesse s'appuie aussi bien sur des données scripturaires que sur des données philosophiques car, pour lui,

«c'est de la même source que proviennent la vérité et l'enseignement de la théologie, de la philosophie naturelle, de la philosophie morale, des savoirs pratiques et théoriques, et même du droit positif, selon dit le Psaume [16,2]: De ta face sortira mon jugement». Eckhart, In Ioh. n.444, LW 3, 381,5-7 (notre trad.)

«Moïse, Christ et le philosophe [Aristote] enseignent donc la même chose. Ils ne diffèrent que par les modes de leur enseignement, à savoir le crédible, le démontrable ou le vraisemblable, et la vérité». In Ioh. n.185, LW 3, 155,5 (OLME 6, 335).

Ainsi, le Thuringien prend position sur les débats universitaires de son temps. En effet, les grecs avaient passé d'une sagesse pratique, attribuée aux artisans comme "excellence de l'art», à la recherche d'une connaissance philosophique, et d'une sagesse comme «science qui concerne les premières causes et les premiers principes» (Aristote). Les stoïciens concédaient aussi une grande importance à l'exercice pratique de la sagesse

[abrégé: OLME 1]; Commentaire de l'évangile selon Jean. Le prologue. L'ouvre latine de Maître Eckhart 6, traduction d'Alain de Libéra et al. Paris: Cerf, 2007 [abrégé: OLME 6]. Pour les sermons latins, La mesure de l'amour. Sermons parisiens, traduction d'Éric Mangin. Paris: Seuil, 2009 [abrégé: EM]; et pour les écrits allemands, Sermons, traités, poème, traduction de Jeanne Ancelet-Hustache, Éric Mangin. Paris: Seuil, 2015 [abrégé: STP]. Mais nous suivons la numérotation des sermons selon l'édition critique, et non pas l'ordre liturgique proposé par Mangin dans STP, à la suite de Loris Sturlese.

4 Cf. Donald F. Duclow. "Meister Eckhart on the Book of Wisdom: Commentary and Sermons". Traditio 43 (1987): 215-235; Andreas Speer. "Weisheit bei Augustinus und Meister Eckhart". Dans Meister Eckhart und Augustinus, édition de Rudolf K. Weigand, Regina D. Schiewer, 1-15. Stuttgart: Kohlhammer, 2011; Marie-Anne Vannier. Maître Eckhart prédicateur. Paris: Beauchesne, 2018, 206-210 et 726. 
comme «science des vertus divines et humaines» (Chrysippe) $)^{5}$, et Boèce désigne la philosophie comme «amour et recherche de la sagesse» (amor vel studium sapientiae). Cette tension entre une compréhension existentielle et une compréhension épistémique de la sagesse, déjà présente dans l'Antiquité, reparaît dans les discussions médiévales sur le statut de la philosophie et son rôle par rapport à la sagesse chrétienne et la vérité de l'Écriture; discussions stimulées avec l'entrée d'Aristote dans le monde latin 6 . Pour sa part, tout en appréciant Aristote — «le philosophe»-, Eckhart souligne l'unité de la vérité (à l'encontre de l'averroïsme latin) et la consonnance de tous les savoirs: pratiques et théoriques, théologie et philosophie, sciences naturelles et éthique.

Pour établir ce qu'il entend par sagesse, nous tiendrons compte spécialement de son Commentaire du Livre de la Sagesse (In Sap. n.121, 201-206, 243 et 272-274), Les sermons et leçons sur l'Ecclésiastique (In Eccli.), et les sermons latins Sermo die b. Augustini Parisius habitus et le Sermon LV, ainsi que les Sermons allemands 95a, 95b et 112, où le Thuringien envisage le sujet plus largement. Nous pouvons commencer par distinguer deux grands volets, la Sagesse divine, c'est-à-dire, la «Sagesse incréée», et la sagesse humaine, la «sagesse participée».

\section{SAGESSE DIVINE - SAPIENTIA INCREATA}

En reprenant les notions qui faisaient partie de l'héritage théologique de son temps, et qui s'appuyaient sur l'Écriture, Maître Eckhart se réfère souvent à Dieu sous le nom de Sagesse: «la Sagesse, laquelle est Dieu» (sapientia, quae deus est) ${ }^{7}$, "la Sagesse ou Dieu» (sapientia sive deus) ${ }^{8}$,

5 Cf. A. Solignac. "Sagesse antique, sagesse chrétienne". Dans Dictionnaire de Spiritualité. Vol. 14, 96-99. Paris: Beauchesne, 1990.

6 Cf. Andreas Speer. "Philosophie als Lebensform? Zum Verhältnis von Philosophie und Weisheit im Mittelalter". Tijdschrift voor Filosofie 62 (2000): 3-25; Andreas Speer. "Sapientia nostra. Zum Verhältnis von philosophischer und theologischer Weisheit in den Pariser Debatten am Ende des 13. Jahrhunderts". En Nach der Verurteilung von 1277. Philosophie und Theologie an der Universität von Paris im letzten Viertel des 13. Jahrhunderts, dirigé par Jan A. Aertsen, Kent Emery, Andreas Speer. Berlin: De Gruyter, 2001, 248-275.

7 Eckhart. Prol. op. prop. n.13, LW 1,1, 173,9 (OLME 1, 81); In Gen. n.159, LW 1,1, 307,2 (OLME 1, 445); In Sap. n.125, LW 2, 463,2 (JCL, 155); In Sap. n.157, LW 2, 493,7 (JCL, 172).

8 Eckhart. In Sap. n.147, LW 2, 485,5; In Sap. n.213, LW 2, 549,6. 
«Dieu Sagesse» (deus sapientia) ${ }^{9}$; «la Sagesse incréée» (sapientia increata $)^{10}$, la «Sagesse divine» (divina sapientia $)^{11}$. La sagesse désigne aussi un attribut ou une perfection de Dieu, comme il affirme dans le Sermon $77^{12}$ ou dans son Commentaire de l'Exode:

«Dieu est son être même. [...] Il ne manque ni de la sagesse ni de la puissance ni de quoi que ce soit de différent ou d'étranger qu'on lui ajouterait, mais inversement toute perfection manque de lui, qui est l'être même». Eckhart, In Ex. n.21 (LW II, 27,8-28,8).

Le Maître thuringien considère également que la sagesse désigne le Verbe, le Fils de Dieu. Il signale qu'en Dieu, on approprie la sagesse et la vérité au Fils ${ }^{13}$. Dans le Commentaire à l'Evangile de Jean, Eckhart nomme le Verbe comme «sagesse incréée» ${ }^{14}$. Et tout comme Origène ${ }^{15}$ et Augustin ${ }^{16}$,

9 Eckhart. In Sap. n.76, LW 2, 407,10; In Sap. n.78, LW2, 410,9; In Sap. n.190, LW 2, 525,11.

10 Eckhart. In Eccli. n.7, LW 2, 235 (B, 18); In Sap. LW 2 n.92, 425; In Sap. n.93, LW 2, 426; In Ioh. n.491, LW 3, 423,5; Sermo 50 n.536, LW 4, 451,5.

11 Eckhart. In Eccli. n.6, LW 2, 234; In Eccli. n.32, LW 2, 261; In Eccli. n.33, LW 2, 263; In Sap. n.78, LW 2, 410,9; In Sap. n.138, LW 2, 476,9 (JCL, 162).

12 Eckhart. Sermon 77, DW III, 341,3 (TSP, 67) «Car la bonté et la sagesse et tout ce que l'on peut dire de Dieu, tout cela est être d'accompagnement (accident) du pur être de Dieu».

13 Eckhart. In Sap. n.89, LW II, 422,8 (JCL, 131) «L'esprit de la Sagesse est venu en moi, c'est-à-dire, l'esprit du Fils, qui est la Sagesse et la Vérité propres dans les réalités divines». Eckhart dit aussi: «la Sagesse divine, le Verbe» (sapientia dei, verbum) dans In Sap. n.243, LW II, 577,4 et n.279, LW II, 611,6; et «la Sagesse divine, le Fils», Sermo 2 n.18, LW IV, 20,6.

14 In Ioh. n.490, LW 3, 422,10 «Eccli. 24 (14): 'ante saecula creata sum' [...] sum ego, scilicet sapientia increata, deus verbum dei».

15 Origène avait dit: «La Sagesse est appelée en troisième lieu le rayonnement de la lumière éternelle. [...] On appelle à proprement parler perpétuel ou éternel ce qui n'a pas commencé d'exister et qui ne cessera pas d'être ce qu'il est. Cela est exprimé par Jean quand il dit que Dieu est lumière [1Jn 1,5]. La Sagesse de Dieu est en effet le rayonnement de sa lumière, non seulement en tant qu'il est lumière, mais aussi en tant que cette lumière est éternelle: ainsi la Sagesse est à la fois éternelle et rayonnement de son éternité. Si on comprend bien cela, il est clair que l'être subsistant du Fils dérive du Père lui-même, mais d'une manière qui n'est pas temporelle, et sans aucun commencement si ce n'est Dieu lui-même». De principiis I, 2, 11 (traduit par H. Crouzel et M. Simonetti. Paris: Cerf [SC 252], 1978, 139).

16 Augustin, De Trin. IV, 20, 27 (traduit par E. Hendrikx, M. Mellet, Th. Camelot. Paris: Desclée de Brower [BA 15], 1955, 407-409) «Par ailleurs, le Fils est le Verbe du Père. On l'appelle aussi sa Sagesse. Quoi d'étonnant alors si le Fils est 
il reprend l'interprétation allégorique des textes du chapitre 7 du Livre de la Sagesse en les appliquant au Fils. Après avoir exposé neuf points sur le Fils comme «image de Dieu» (In Ioh. n.23-26), Eckhart conclut ceci:

«Or ce qu'on a dit de l'image est clairement résumé au chapitre septième [du Livre] de la Sagesse, où il est dit de la Sagesse ou Verbe de Dieu qu'elle est un miroir sans tache [Sg 7,26b], une émanation toute pure de Dieu [Sg 7,25b], qu'elle est aussi une image de sa bonté [Sg 7,26c] et que rien de souillé ne pénètre en elle [Sg 7,25c]; et encore qu'elle est un souffle de la puissance divine [Sg 7,25a] et l'éclat de la lumière éternelle $[\operatorname{Sg} 7,26 \mathrm{a}]$.

Comme on l'a dit plus haut, c'est également ainsi qu'on interprète quasiment tout ce que dit l'Ecriture sur la divinité du Fils». In Ioh. n.27 (OLME 6, 65).

Finalement, Eckhart attribue aussi la sagesse à Jésus-Christ, et il se sert à plusieurs reprises de certains textes du Nouveau Testament qui font allusion à la sagesse du Christ (Verbe incarné). Ainsi il emploie trois fois l'épitre aux Corinthiens (1Cor 1,24) «le Christ puissance de Dieu et la sagesse de Dieu (dei virtus et dei sapientia)» ${ }^{17}$; et Colossiens ( $\left.\mathrm{Col} 2,3\right)$, selon la leçon de la Vulgate ${ }^{18}$, qui spécifie que c'est du Christ dont on parle, «en qui sont cachés tous les trésors de la sagesse et de la science» (In Gen. n.301 et Sermon XXII n.201). Également, il applique au Christ le texte de l'Ecclésiastique 1, $5^{19}$ et il dit dans son Sermon XXIV,1 n.227: «Note: notre Sauveur et modèle [est] la source de la sagesse, le Verbe de Dieu, au plus haut [des cieux], Sir 1", affirmation qu'il reprend dans le Commentaire de l'évangile selon Jean: «Moi [le Christ] qui suis source de la sagesse, Sir $1 »^{20}$.

envoyé, non qu'il soit inférieur au Père, mais parce qu'il est une émanation pure de la clarté du Dieu tout puissant (manatio qucedam est claritatis omnipotentis Dei sincera) $(\mathrm{Sg} 7,25 \mathrm{~b})$ ? Ici ce qui émane et ce dont il émane sont une seule et même substance. [...] Il n'y a pas non plus à s'émouvoir de la voir appeler une émanation pure de la clarté du Dieu tout puissant (Sg 7,25b), en ce sens qu'elle-même ne serait pas toute puissante, mais émanation du tout puissant. Le texte ajoute aussitôt, en effet, étant unique, elle peut tout ( $\operatorname{Sg} 7,27 \mathrm{a})$. Or qui est toute puissante sinon celle qui peut tout».

17 Eckhart. In Eccli. n.4, LW 2, 233,3; In Sap. n.89, LW 2, 422,9; Sermon 106, DW $4,684,15$.

$18 \mathrm{Col}$ 2,3: Christi Jesu: in quo sunt omnes thesauri sapientice et scientice absconditi.

19 Sir 1,5: Fons sapientice verbum Dei in excelsis.

20 Eckhart. In Ioh. n.671, LW 3, 584,8 «ego qui 'fons' sum 'sapientiae', Eccli. 1 ». 
Malgré notre effort de classification des notions eckhartiennes de la sagesse, force est de constater qu'Eckhart ne se préoccupe pas de délimiter les différents sens de la sagesse, peut-être du fait qu'il n'apprécie guère les divisons et les particularités, et il tend toujours vers l'unité. Ainsi, dans les Sermons et leçons sur l'Ecclésiastique, le Thuringien parle de «la Sagesse incréée de Dieu» (sapientia dei increata) (In Eccli. n.7, B, 18), qui peut aussi bien se référer à Dieu «sous l'espèce et la forme de la Sagesse» (deus, sub specie et forma sapientiae) $)^{21}$ que se référer à l'une des Personnes de la Trinité: «la sagesse de Dieu le Père» (sapientia dei patris) 22 ou bien au Fils, lui aussi «émanation pure de Dieu» ${ }^{23}$. Et il invoque également «la Sagesse incarnée» (sapientia incarnata) ${ }^{24}$, donc Jésus-Christ, qu'il désigne aussi comme Sagesse ${ }^{25}$. Et il arrive même à passer d'un sens à l'autre dans une même phrase, en entendant la sagesse comme Dieu ou sa nature, comme le Verbe ou comme le don de Dieu (Sermon LV n.536).

Une fois signalés les différents sens de la sagesse divine pour Eckhart, il est intéressant d'approfondir quelques caractéristiques de celle-ci. Étant donné que le Thuringien commente des textes bibliques sapientiaux où la sagesse apparaît personnifiée, les allusions à celle-ci sont nombreuses, et il est fréquent qu'il commence à parler de «la Sagesse divine» (divina sapientia) ou de «Dieu Sagesse» (deus sapientia) et qu'il se réfère plus tard à Dieu tout court. Mais nous pouvons dégager aussi certains aspects de la Sagesse divine en tant que telle. Ainsi, dans son premier Sermon sur l'Ecclésiastique, Eckhart explique ceci:

«La Sagesse divine nous invite aujourd'hui en disant dans l'épitre: 'Passez tous à moi' [Sir 24,23]. En commençant elle promet trois choses que tous ont coutume d'aimer, ni plus, ni moins, ni aucune autre. Ce sont: la pureté de l'aimé, sa plénitude ou son abondance, et troisièmement sa suavité» ${ }^{26}$.

21 Eckhart. In Eccli. n.51, LW 2, 280,3 (B, 51).

22 Eckhart. In Eccli. n.39, LW 2, 266,8 (B, 40).

23 Eckhart. In Eccli. n.8, LW 2, 237,7s (B, 19) «le Fils, lequel est une émanation formelle excluant l'efficience et la fin, selon ces paroles: (La Sagessse) est l'émanation pure de Dieu, Sagesse 7 [25b]».

24 Eckhart. In Eccli. n.14, LW 2, 243,10 (B, 23); et aussi In Eccli. n.7 «la Sagesse même de Dieu s'est incarnée miséricordieusement» $(\mathrm{B}, 18)$.

25 Eckhart. In Sap. n.124, LW 2, 462,4 (JCL, 154) «la Sagesse [le Christ] fait des reproches, en Jean 8, (49): Vous, vous me déshonorez,.

26 Eckhart. In Eccli. n.6, LW 2, 234,12-235,3 (B, 18). Dans un deuxième sermon sur Sir 24,27, Eckhart offre cinq caractéristiques de la sagesse: la pureté, sa propriété 
Ce sont les trois points que nous allons retenir: la pureté de la Sagesse (puritas), son abondance qui est source de toutes les choses, ce qui fait qu' «elle est mère de tous les biens» $(\mathrm{Sg} 7,12)$, et sa douceur (suavitas).

\subsection{La PURETÉ de La SAgesse}

En premier lieu, la sagesse renvoie à «la pureté sans mélange de l'essence divine» (divinae essentia impermixta puritas) (In Eccli. n.34, LW 2, $262,12, \mathrm{~B}, 37)^{27}$. Il est clair que les commentaires eckhartiens sur la sagesse sont aussi des développements de son enseignement sur Dieu. Ainsi, le Maître thuringien explique que «quand on dit de la Sagesse qu'elle est une, on montre la plénitude et la pureté de son être, de même que sa bonté [...et] la suprême et primordiale perfection de Lui-même» (In Sap. n.145, JCL, 166). L'unité de Dieu implique aussi son indistinction de toutes les choses, «soit parce qu'il est infini, soit parce qu'il n'est pas circonscrit aux limites [...] d'un étant» (In Sap. n.144, JCL, 165). C'est pourquoi Eckhart peut soutenir que «Dieu est aussi bien totalement en dedans de toute chose, que totalement en dehors de toute chose» (In Sap. n.135, JCL, 161). Par son indistinction, Dieu n'est pas limité par les choses mais, en même temps, c'est cette indistinction qui le rend absolument distinct de tout ce qui est en tant que 'ceci' et 'cela' (hoc et hoc). Il s'agit donc de l'immanence divine ainsi que de sa transcendance. Le Thuringien élargit sa réflexion sur la pureté de l'être, qui correspond à l'unité. Comme l'unité signifie la négation de toute division (division qui, en elle-même, suppose une négativité), il soutient avec le Pseudo-Denys ${ }^{28}$, Jean Damascène ${ }^{29}$ et Albert le Grand ${ }^{30}$ que la «négation de la négation, est l'affirmation la plus pure et la plénitude du terme affirmé», plénitude qui convient à l'un (In Sap. n.147) ${ }^{31}$.

de source du bien universel, son excellence, sa perfection et la suavité (In Eccli. n.33, LW 2, 262,7-11).

27 Cf. Vannier. Maître Eckhart prédicateur, 207.

28 Ps.-Denys. De myst. theol. III-V. Dans Dionysiaca, édition de Philippe Chevallier. Vol. 1. Paris: Desclée de Brower, 1937, 589-602.

29 Johannes Damascenus. De fide orthodoxa I 4, édition d' Eligius M. Buytaert. New York: Franciscan Institute Publications, 1955, 20-21.

30 Albertus Magnus. Super Mysticam theologiam Dionysii, edition de Paul Simon. Münster: Aschendorff, 1978, 471.

31 Cf. Jan A. Aersten. "La doctrine des transcendantaux de Maître Eckhart". Dans Maître Eckhart, édition de Julie Casteigt, 28-29. Paris: Cerf, 2012. 
Eckhart expose aussi le verset Sg 7,24b «Elle s'étend partout à cause de sa pureté» et il le relie à $\mathrm{Sg} 7,25 \mathrm{c}$ «Rien de souillé ne pénètre en elle», comme le faisait aussi Saint Augustin ${ }^{32}$, sur lequel il s'appuie. Un des sens du texte, signale le Dominicain, est que la sagesse s'étend partout pour se communiquer et rendre pures toutes les choses.

Maître Eckhart affirme aussi que «toute chose est dite pure, lorsque rien d'étranger ne se retrouve en elle» (In Sap. n.137, JCL, 162). C'est bien le cas de «Dieu, en tant que Très Haut (supremum), il touche et affecte tout ce qui est plus bas que lui, mais il n'est touché ni affecté par ce qui est plus bas» (In Sap. n.134). C'est un principe néoplatonicien cher au Thuringien, qui le répète souvent: le supérieur affecte l'inférieur, mais il n'en est pas affecté par celui-ci. En revanche, il est principe d'unité de l'inférieur ${ }^{33}$. Car, «Dieu porte son regard et atteint toutes choses et tous lieux, quant à leurs essences, immédiatement et par Lui-même. La raison en est que Dieu est l'être pur et plein. Or l'être ne considère et ne connait absolument rien d'autre que l'essence des choses» (In Sap. n.140, JCL, 163). Et ce regard de Dieu est celui qui leur donne l'être, et leur confère leur forme. Ceci nous conduit au second aspect, «la plénitude et l'abondance» de Dieu-Sagesse, qui «témoigne sa fécondité» (In Eccli. n.11, B, 21).

\subsection{L’abondance de la Sagesse: «elle est mère de tous les biens» (Sg 7,12$)$}

Eckhart commente aussi le verset $\mathrm{Sg} 7,12$, Elle est mère de tous les biens, verset qui n'a jamais été cité par Augustin ${ }^{34}$, bien que les réflexions eckhartiennes à son sujet retrouvent la pensée augustinienne de la création continuée. Le Thuringien va approfondir le sens de cette maternité de la Sagesse; elle a trois caractéristiques: la réception, la contenance et la conservation. Ainsi, Eckhart affirme:

«Il faut remarquer par conséquent que le texte dit: la mère de tous les biens. Il ne dit pas: 'le père', bien que cela soit vrai aussi; il préfère dire la mère. Un enfant, en effet, bien qu'il provienne de façon active du père, est cependant jusqu'à dix mois durant [...] dans la mère sur

32 Cf. l'index d'Anne-Marie La Bonnardière. Biblia Augustiniana. AT. Le Livre de la Sagesse. Paris: Études Augustiniennes, 1970, 282.

33 Dans In Sap. n.39, LW 2, 360,11, Eckhart renvoie à Proclus, Eléments de théologie, prop. 1.

34 Il n'apparait pas dans l'index de La Bonnardière, 281. 
le mode de la réception, de la contenance et de la conservation. Le Sage [l'auteur du Livre de la Sagesse] veut donc dire que tous les biens, et aussi toutes les choses créées, non seulement viennent de Dieu qui crée activement, mais aussi sont en Dieu sur le mode de la contenance et de la conservation». In Sap. n.121 (JCL, 152).

Dieu, en tant que sagesse, non seulement crée les choses, mais les maintient et conserve. Eckhart justifie son affirmation par l'autorité de l'Écriture, car les psaumes disent bien: «Tu as tout fait dans la Sagesse» (Ps 104 (103),24) et «avec intelligence» (Ps 135,5); mais aussi par l'autorité d'Augustin qui soutient, dans ses Confessions IV, 12, 18, que «Dieu fit toutes choses; mais "il ne le fit pas pour les abandonner, mais parce qu'elles viennent de Lui, elles sont en Lui”»(In Sap. n.121) ${ }^{35}$. Et il continue son explication: Dieu ne crée pas toutes les choses «en dehors de Lui», mais «à partir de Lui et en Lui», car «ce qui est hors de Dieu est hors de l'être» et «il n'y a rien en dehors de Lui». Et cela «rejoint Romains 11 (36): Toutes choses sont de Lui, avec Lui et en Lui»(In Sap. n.122, JCL, 153). Il s'agit donc de la contenance et de la conservation, la création continuée de Dieu. Le Thuringien développe ces mêmes pensées dans le Prologue général à l'Euvre Tripartite n.16-19. "La création est la collation de l'être» (collatio esse) (Prol. gen. n.16), et «Dieu a créé au commencement, c'est-à-dire en lui-même», car «avant lui et en dehors de lui, il n'y a rien» (n.17). Et «Dieu a créé toutes choses, non pour qu'elles se tiennent en dehors de lui, à côté de lui ou en plus de lui, à la manière des autres artisans, mais il les a appelées du néant, c'est-à-dire du non-être, à l'être qu'elles trouveraient, recevraient et posséderaient en lui. Car il est l'être» (Prol. gen. n.17). Eckhart s'inspire aussi d'Augustin pour soutenir que Dieu a créé "de telle sorte qu'il crée toujours» (Prol. gen. n.18). Il cite explicitement cette phrase du premier livre des Confessions I, 6, 10: «Toutes les choses d'hier et du passé, tu les feras aujourd'hui».

Finalement, dans le Sermon allemand 40, Maître Eckhart reprend l'aspect maternel de la Sagesse, sous l'angle de la réception. Ainsi, il y

35 Eckhart. In Sap. n.121, LW 2, 458,9-10. Saint Augustin, Conf. IV, 12, 18 (édition de A. Solignac, E. Tréhorel, G. Bouissou. Paris: Desclée de Brower [BA 13], 1962, 438) "Non enim fecit atque abiit, sed ex illo in illo sunt». Eckhart apprécie beaucoup cette idée augustinienne, car il la reprend à 9 occasions tout au long de son œuvre: Prol. gen. n.17, n.20 et n.21, LW 1/1, 161,6; 164,9; et 165,4; In Gen. n.19, LW 1/1, 201,2; In Eccli. n.49, LW 2, 278,2; In Sap. n.121, 458,8; In Ioh. n.579, 508,2; Sermo XXIII, LW IV, 207,14; et Sermon allemand 30, DW 2,101,1. 
signale que «Sagesse est un nom maternel, car un nom maternel désigne la propriété de souffrir» (TSP, 451). 'Souffrir', dans le sens de recevoir, de subir, eines lîdennes, en moyen-haut allemand. En Dieu, dans la vie intratrinitaire, «sont l'opération et la souffrance» (wirken und lîden), car le Père est actif ou opérant, et le fils est passif ou souffrant. «Il en est ainsi en raison de la propriété de la naissance. Voilà pourquoi —conclut Eckhart-, le Fils est la sagesse qui naît éternellement» (TSP, 451). Il est 'sagesse', car il est passif et réceptif, et il nait éternellement car, en Dieu, le Fils nait toujours et l'action ne passe jamais au passé, comme remarquait Origène dans son Homélie sur Jérémie 11, texte qui a profondément marqué le Maître thuringien ${ }^{36}$. Ainsi, il le reprend souvent:

«En effet, en Dieu même, le Père n'aurait pas engendré le Fils, si avoir engendré n'était pas engendrer. C'est pourquoi Origène (dans son commentaire) sur Jérémie fait dire à la personne du Fils: "Le Seigneur m'engendre avant toutes les collines", Prov. 8, et non pas "m’a engendré", selon la mauvaise leçon de certains. (Ce sont) les paroles d'Origène». In Eccli n.23 (B, 28).

Et ailleurs il rappelle: "Origène dit, dans la Glose sur Jérémie 11,(9): "à chaque acte de vertu" un homme "naît fils de Dieu" » ${ }^{37}$. C'est pourquoi, plus les humains sont vertueux et plus ils deviennent réceptifs et se détachent de tout, plus ils permettent que l'image de Dieu, en laquelle ils sont créés, luise en eux. Ils deviennent alors fils de Dieu et se conforment à cette sagesse passive, qui reçoit tout et constamment du Père.

\subsection{La Suavité de la SagesSe: «Elle dispose aVec Douceur toutes Les Choses»} $\left(S_{G} 8,1\right)$.

Cette notion de suavité et de douceur de la Sagesse a une grande importance pour Eckhart, car dans son Commentaire du Livre de la Sagesse, il déploie 22 raisons pour exposer comment «l'action de Dieu, et de Lui

36 Cf. Elisabeth Boncour. "La source origénienne de la naissance du Verbe en l'âme et le traitement eckhartien”. Dans Maître Eckhart, lecteur des Pères grecs, dirigé par Marie-Anne Vannier. Paris: Beauchesne, 2020, 43-57. Eckhart cite explicitement onze fois un fragment de l'Homélie sur Jérémie 11 d'Origène, qui parle de la naissance éternelle et incessante du Fils en Dieu et en chaque personne vertueuse, et qu'il connait à travers la Glose ordinaire.

37 Eckhart. Par. Gen. n.191 (JCL, 204). Origène, Hom. in Jer. 11. 
seul, est douce pour les créatures»(In Sap. n.173, JCL, 183) ${ }^{38}$. Et ceci s'applique à Dieu sous le nom de Sagesse, car «il revient, en effet, aux sages d'ordonner l'action, comme il est dit dans le premier livre de la Métaphysique [d'Aristote $]^{39}$, en vue d'une fin et par les moyens intermédiaires adaptés à cette fin» (In Sap. n.186). Et Dieu «dirige les choses singulières vers leurs fins propres, par des moyens en accord avec les fins propres de chacune» d'elles, explique le Maître thuringien. En fait, «l'action de Dieu dans les créatures est elle-même la fin, et en même temps la disposition» des choses (In Sap. n.188, JCL, 193). Dieu donne aux choses leur «être» (In Sap. n.177, JCL, 185), leurs «formes et leurs caractères propres» (In Sap. n.173, JCL, 183), et alors leurs penchants naturels sont doux pour elles, car il y a un accord entre l'acte et la disposition innée, ce qui donne lieu à une douceur, une délectation. Eckhart renvoie au livre $\mathrm{X}$ des Confessions de Saint Augustin, où il se dirige ainsi à Dieu: «Donne ce que tu ordonnes, et ordonne ce que Tu veux» ${ }^{40}$ (In Sap. n.173, JCL, 183). Le Dominicain approfondit l'affirmation augustinienne dans un sens ontologique, et il est loin de vouloir opposer la nature et la grâce. "Il est naturel pour l'ensemble des choses créées et pour chacune d'elles —explique-t-ilque Dieu veuille agir et agisse en elles. Or tout ce qui est naturel pour les choses, leur est doux et suave» (In Sap. n.180, JCL, 187). Toute action qui vient de l'intérieur, poursuit Eckhart, est agréable et douce, car en elle il y a un accord entre l'acte et l'aptitude (actus et habitus) (In Sap. n.184). C'est une caractéristique de "la vie ou du fait de vivre», car «le vivant se meut de lui-même et du dedans». (In Sap. n.184, 190). Alors:

«Dieu, en tant que cause première et fin dernière, est intérieur à toutes les choses qu'il met en mouvement. De là vient que toutes les opérations, et elles seules, que Dieu réalise en nous et nous en Lui, pour Lui en tant que plus intérieur, sont vives. Mais toutes les actions, que nous réalisons pour quelque chose d'autre en dehors de Lui, —qui est pour nous véritablement intime, qui pénètre dans l'essence, en tant qu'être-, sont mortes, étant donné que dans des actions de ce genre, ce n'est pas Lui qui nous met en mouvement, mais quelque chose d'extérieur, comme quelque chose de mort, qui n'est déjà plus en vie. Car, rien en dehors de Dieu ne pénètre notre essence. [...]

\footnotetext{
38 Selon Donald Duclow, dans son Commentaire du Livre de la Sagesse Eckhart développe sa pensée plus librement que dans In Gen., In Ex. et In Ioh. où il suit les commentaires de Thomas et d'autres auteurs. Cf. Duclow, 219.

39 Aristote, Métaphisique I 2 982a17.

40 Augustin, Conf. X, 29, 40 et 37, 60 «Da quod iubes et iube quod vis».
} 
S'il est vrai que seules ces actions-là nous profitent, c'est qu'elles sont nôtres et divines, et qu'elles viennent du dedans, et c'est parce qu'en elles c'est notre Dieu intérieur au plus haut point qui nous met en mouvement, et rien du dehors ou de l'extérieur». In Sap. n.184, (JCL, 190-191).

Cette explication est forte de sens, et permet de mieux situer certains textes eckhartiens difficiles à comprendre (car il n'est pas toujours facile de savoir s'il parle de la nature ou de la grâce, ou des deux ${ }^{41}$. En fait, c'est Dieu qui a disposé par la création qu'Il soit Lui-même le plus intérieur à nous, et que nos actions en Lui et pour Lui soient douces, tout aussi comme celles qu'il réalise en nous. Alors le désir de Dieu et la capacité de le recevoir et de l'aimer, et toutes nos actions envers Lui sont donc à la fois «nôtres et divines», le fruit de l'action de Dieu en nous et de notre nature qu'il nous a offert. Au fond, tout est un don, tout est grâce ${ }^{42}$ : la grâce de la création qui nous donne d'être ce que nous sommes, capables de Dieu (In Ioh. n.610). C'est ce qu'Eckhart appelle la "grâce donnée gracieusement» (gratia gratis data) (In Sap. n.272, JCL, 248). Le Thuringien remarque que «tout ce que Dieu opère dans la créature est grâce et donné gracieusement» (In Sap. n.272). Mais il souligne que la grâce agit dans l'essence, dans ce que l'on est, mais l'opération, elle, elle nous revient. «La grâce, à proprement parler, ne commence pas l'action, mais concerne l'être à l'intérieur, comme l'essence même, en tant qu'essence, concerne l'être seulement». (In Sap. n.273, JCL, 248). C'est pourquoi nous sommes libres de commencer les actions, et celles-ci sont bien nôtres, et aussi divines, car nous agissons selon ce que Dieu a mis en nous.

Eckhart précise davantage comment se réalise cette action divine en douceur dans les créatures, et il emploie des catégories aristotéliciennes. C'est à travers «la forme substantielle» (forma substantialis) qu'elles ont reçu de lui: «sans murmure ni contradiction, ni résistance de la matière, ni intermédiaire», la forme "demeure et informe la matière avec douceur» (In Sap. n.189). «Or Dieu, la Sagesse, est l'actualisation (actualitas)

${ }^{41}$ Certains semblent oublier cette double dimension divine et humaine chez Eckhart. Ainsi, Speer soutient que selon Eckhart, «l'être humain participe immédiatement de la Sagesse divine, en vertu de sa nature rationnelle». Alors, la recherche de la sagesse s'adresse à «l'homme intérieur, qui, en se retournant sur lui-même, devrait retrouver ce qu'il est par nature». Cf. Speer. "Weisheit bei Augustinus und Meister Eckhart", 13 et 15.

${ }^{42}$ Cf. Édouard-H. Wéber. "La théologie de la grâce chez Maître Eckhart ». Revue des sciences religieuses 70/1 (1996): 48-72. 
elle-même et la forme de tous les actes et de toutes les formes». C'est-à-dire, c'est en Lui et de Lui que viennent toutes les formes dans les choses. Eckhart allègue explicitement l'autorité d'Augustin, dans La Vraie religion 18, 36: «Tout vient de Dieu, ce qui est formé, en tant que formé, et tout ce qui n'est pas encore formé, en tant que cela peut être formé» ${ }^{43}$.

\section{SAGESSE DANS LES HOMMES - SAPIENTIA PARTICIPATA}

Si la Sagesse désigne Dieu même, son essence et spécialement le Fils, le Christ, il y a aussi une dimension «subjective», la sagesse en l'homme, qu'Eckhart appelle «sagesse participée» (In Sap. n.92). Car, pour lui, la sagesse en tant que telle est incréée et incréable, comme il ressort de sa première Question parisienne, où il écrit explicitement que la sagesse appartient à l'intellect et ne fait pas partie de ce qui peut être créé: $S a$ pientia autem, quae pertinet ad intellectum, non habet rationem creabilis (Qu. Par. I n.4, LW 5, 41,10-11). À cet égard, la sagesse en l'homme n'est pas de nature créaturelle au sens propre, mais communiquée par Dieu.

\subsection{La Sagesse de Dieu, par Dieu, dans les hommes}

Déjà Saint Augustin avait exprimé dans son De Trinitate que si l'on parle de sagesse humaine, c'est d'une sagesse telle qu'elle soit en même temps sagesse de Dieu. C'est alors la vraie sagesse; «et ce n'est pas par sa propre lumière, mais par participation à cette lumière suprême qu'elle [l'âme] deviendra sage » ${ }^{44}$. Également, Eckhart est convaincu que la source de toute sagesse et de toute vérité est extérieure aux hommes: elle vient de Dieu, tout comme leur être. C'est pourquoi, quand il expose le verset Sg 13,1: Ils sont vains tous les hommes en qui ne subsiste pas la science de Dieu, il affirme ceci:

"De même que tout "être formel" (ens formale), dans les choses à l'extérieur, est par l'être de Dieu Lui-même, "par lui et en lui" [Rm. 11,36], de même toute vérité ou science est dans les hommes par Dieu Lui-même, en

\footnotetext{
43 In Sap. n.189, LW 2, 524,10-525,4 (JCL, 193).

44 De Trin. XIV, 12, 15 (édition de Paul Agaësse, Joseph Moingt, Paris: Desclée de Brower [BA 16], 1955, 387).
} 
tant que Vérité et Sagesse, suivant Jean 14 [26]: Il vous enseignera toutes choses, et à nouveau, Jean 16 [13]: Il vous enseignera toute la vérité, et Ecclésiastique 1 [1]: Toute sagesse vient du Seigneur Dieu» ${ }^{45}$.

En premier lieu, le Maître thuringien associe «sagesse», "vérité» et «science», mais il ne fait pas de distinction entre science des choses humaines et science des choses divines, comme Augustin (De Trin. XIV, 1, 3); il considère que toute science, de n'importe quelle sorte, et toute vérité viennent de Dieu. Ainsi, Eckhart s'appuie aussi sur Saint Ambroise qui déclare «qu'il importe peu de savoir qui dit la vérité, car elle vient toujours de l'Esprit Saint» ${ }^{46}$. C'est une profonde conviction qu'Eckhart partage et applique: il se sert aussi bien des philosophes grecs et arabes, de penseurs juifs (Maimonide) ou chrétiens, même si Augustin reste l'auteur qu'il cite le plus; et, nous l'avons dit, il met en relation la nature, la philosophie, la théologie et l'éthique, car il considère que toute vérité vient de de la même source, de Dieu ${ }^{47}$. Et donc, pour lui, tous les savoirs sont utiles et recherchent la Vérité.

En deuxième lieu, le texte précédent (In Sap. n.242) invoque «l'être formel» des choses, reçu de Dieu. Dans son Commentaire de la Genèse, le Thuringien explique que «toute créature a un être double». L'un est «celui des choses à l'extérieur, dans la nature»: c'est leur "être formel» (esse formale), l'être que les choses ont «dans leur forme propre», qui informe la matière. "La plupart du temps il est inconsistant et variable», car les choses changent et sont corruptibles. Mais l'autre être est leur «être virtuel» (esse virtuale), c'est-à-dire, l'être qu'elles ont dans leurs causes originaires, en tant qu'elles sont «dans le Verbe de Dieu, et c'est un être fixe et stable» (In Gen. n.77, OLME 1, 334). Dans son Livre des paraboles de la Genèse il signale ainsi l'être double des choses: d'un côté, «les idées qui sont dans l'esprit divin" (ideae in mente divina) ou leur "être intellectuel» et, de l'autre, "l'être naturel au sein de la matière en train de changer ${ }^{48}$. Il s'agit de deux perspectives, l'être des choses selon leur forme créée ou bien prises dans leur cause originaire, in mente divina.

45 Eckhart. In Sap. n 242, LW 2, 576,1s (JCL, 227, trad. modifiée).

46 Ambroise de Milan. Commentaire de la première Épitre aux Corinthiens I, 12, 3.

47 Cf. Eckhart. In Ioh. n.185, LW 3, 154,14-155,2 (OLME 6, 335); y n.444, LW 3, 381,5-7.

48 Eckhart. Par. gen. n.202, LW I,1, 674,4-5 (JCL, 211). 
Le Thuringien désigne aussi cet «être virtuel» comme «raison» $(\text { ratio })^{49}$ ou modèle (exemplar) des choses, et c'est également ce qui permet de les connaître, car il brille en elles. C'est intéressant de souligner que ce n'est pas la raison qui est 'dans' la chose, mais inversement, la chose qui est 'dans' la raison, son principe, qui est antérieur à la chose même (In Eccli n.38). Cette considération est constante chez Eckhart: les choses sont toujours 'dans' leurs principes et donc toute la création en Dieu ${ }^{50}$. Plus concrètement, les raisons de toutes les choses sont comprises dans le Verbe, comme il le dit à plusieurs reprises ${ }^{51}$, en s'appuyant explicitement sur le De Trinitate VI, 10, 11 de Saint Augustin ${ }^{52}$.

Le Thuringien recours également à une œuvre augustinienne pour exprimer cette double dimension, les deux mondes de Platon:

"Ce qu'Augustin dit (dans son traité) Contre les académiciens [III, 17,37 s se rapporte à ce qui précède: "Platon soutenait qu'il y avait deux mondes: l'un intelligible, dans lequel habite la vérité, l'autre, sensible, qui est manifeste au sens par la vue et le toucher. Celui-là est véritable, celui-ci est vraisemblable et fait à l'image du premier" ${ }^{53}$.

"Augustin dit, d'après Platon, que la sagesse et la vérité ne sont pas dans ce monde, mais dans un monde plus élevé, à savoir le monde intellectuel» ${ }^{54}$.

Par conséquent, Eckhart situe la vérité et la sagesse dans un monde au-delà du sensible, le monde intelligible, qui appartient à la sphère divine. Platon n'est pas l'unique source eckhartienne, il intègre également les principes néoplatoniciens du Livre des causes (extrait des Éléments de théologie de Proclus) ainsi que la Métaphysique d'Aristote ${ }^{55}$.

49 Jan Aersten pointe que, dans les considérations d'Eckhart, le terme ratio n'est pas seulement «le concept intelligible d'une chose dans l'intellect humain, mais l'idée exemplaire dans la pensée divine». Cf. Aersten, 31.

50 Principe qui s'appuie sur le Livre des causes, prop. 19 n.150, dans Le 'Liber de causis'. Édition établie à l'aide de 90 manuscrits avec introduction et notes, édition de A. Pattin. Leuven, 1967, 89, 97-98.

51 Cf. Eckhart. In Gen. n.5 (OLME 1, 245) «Dans le commencement, c'est-à-dire, dans le Fils, qui est le modèle et la raison idéelle de toutes choses»; In Sap. n.189 (JCL, 193) «Dieu, la Sagesse, est l'actualisation elle-même et la forme de tous les actes et de toutes les formes».

52 Cf. Eckhart. In Ioh. n.15, LW 3, 12 (OLME 6, 47).

53 Eckhart. In Gen. n.78, LW 1,1, 239,8-11 (OLME 1, 337-339).

54 Eckhart. In Eccli. n.10, LW 2, 240,5 (B, 21). Cf. Augustin, C. acad. III, 7, 37.

55 Les sources eckhartiennes sont nombreuses, il intègre le platonisme et néoplatonisme augustinien avec le néoplatonisme dionysien, Proclus et le Liber de causis, 
L’originalité de la pensée éckhartienne par rapport à la sagesse apparait clairement dans le Prologue général de l'Opus tripartitum, où Maître Eckhart annonce le plan complet de son œuvre, ainsi que les concepts fondamentaux (termini generales) sur lesquels il fonde sa pensée et qu'il développera en quatorze traités, dans son Euvre des propositions. Parmi ces termini il inclut non seulement les transcendantaux traditionnels (être, unité, vérité, bonté) mais aussi les «perfections spirituelles»" ${ }^{56}$, comme la sagesse (tout comme Duns Scott) et la justice, qu'il classe parmi les «perfections les plus générales». Ainsi le Thuringien précise:

"On ne doit en aucune façon se représenter ou juger les termes généraux -comme être, unité, vérité, sagesse, bonté et les autres semblables- d'après le mode et la nature des accidents qui reçoivent l'être dans le sujet, par le sujet, et par son changement, sont postérieurs à lui et reçoivent l'être par inhérence. [...]

Or, il en va tout autrement des termes généraux susdits. En effet, l'être lui-même et ce qui, se convertissant avec lui, est la même chose que lui, ne surviennent pas aux choses comme postérieurs, mais sont antérieurs a tout dans les choses. Car l'être lui-même ne reçoit pas le fait d'être ni en quelque chose, ni de quelque chose, ni par quelque chose; et il n'advient ni ne survient à quelque chose, mais il (le) prévient et il est antérieur à tout. C'est pourquoi l'être de toutes les choses est immédiatement par la cause première et par la cause universelle de toutes choses. Donc, de l'être lui-même, 'par lui et en lui sont toutes choses' [Rom. 11,36], \$7.

Ainsi, selon Eckhart, les propriétés générales, comme la sagesse ou la justice, ainsi que les transcendantaux, l'être, l'unité, la vérité, la bonté, sont antérieures aux choses et les rendent possibles. Elles se trouvent et appartiennent au sens propre à Dieu, cause première et universelle de toutes les choses. L'identification des transcendantaux (et des termini generales) à Dieu est une des péculiarités de la pensée du Thuringien ${ }^{58}$.

C'est pourquoi «elles se disent de Dieu et de la créature analogiquement» ${ }^{59}$, comme l'explique Eckhart dans ses Sermons sur l'Ecclésiastique

Aristote, Thomas, Avicenne, Maimonide, Origène, etc. Cf. Loris Sturlese, ed. Studie sulle fonti di Meister Eckhart, 2 vols. Fribourg: Dokimion 34 et 37, 2008 et 2013.

56 Eckhart. In Sap. n.41, LW 2, 363,9-10.

57 Eckhart. Prol. gen. n.8, LW 1,1, 152,8-153,6 (OLME 1, 49).

58 Cf. Aersten, 34-36.

59 Eckhart. In Eccli. n.52, LW 2, 280,5-281,12 (B, 51). Ainsi, dit Eckhart, «les équivoques se divisent selon les diverses choses signifiées, mais les univoques selon les différences de la chose, tandis que les analogues ne se distinguent pas selon les 
n.53: «Tout étant créé est analogué à Dieu dans l'être, la vérité et la bonté». Mais dans les choses créés, c'est-à dire, «dans les analogués, il n’y a aucun enracinement positif de la forme à laquelle ils sont analogués» ${ }^{60}$. Les 'étants' —et tous les autres termes généraux, une fois concrétés, comme le vrai ou le juste- ont "par Dieu et en Dieu», et non dans leur être créé, leur être, vérité, justice ou sagesse.

La portée de l'analogie chez Eckhart reste un sujet à débat, certains signalent qu'il ne semble retenir que l'analogie dite 'd'attribution' ${ }^{61}$ —avis que nous partageons - et d'autres, comme Mojsisch, l'identifient avec l'univocité ${ }^{62}$. En tout cas, le Thuringien ne considère donc pas que l'être ou la vérité et les termes généraux soient des concepts tirés à partir des choses (par abstraction) et postérieurs à elles, mais qu'ils sont antérieurs aux choses. Ainsi ils précèdent la réalité empirique, ils sont la condition de possibilité de cette réalité et aussi de sa connaissance.

Dans le Prologue général, Eckhart justifie ses réflexions en s'appuyant sur Saint Augustin:

"Augustin, De la Trinité VII, chap. 1 (2), faisant allusion à ce qui précède, déclare: "La sagesse ... est sage et elle est sage par elle-même. Et toute âme devient sage par sa participation à la sagesse; si elle retombe dans la folie, la sagesse demeure cependant en soi et ne change pas non plus quand l'âme a changé en déraison. En celui qui est sage par la sagesse, ce n'est pas comme la blancheur dans le corps qui est blanc par elle. Car, lorsque le corps aura passé à une autre couleur, cette blancheur ne demeurera pas et cessera absolument d'être" ${ }^{63}$.

Eckhart va utiliser constamment cette relation sage / sagesse ou, plus souvent, juste / justice ${ }^{64}$, pour mettre en évidence, d'une part, que ce que

choses, ni non plus selon les différences des choses, mais ‘selon les modes' d'une seule et même chose absolument».

60 Eckhart. In Eccli. n.53, LW 2, 282,1-3 (B, 52).

61 Cf., par éxemple, Fernand Brunner. "Lanalogie chez Maître Eckhart". Freiburger Zeitschrift für Philosophie und Theologie 16 (1969): 333-349; A. Schiffhauer. "nos filii dei sumus analogice. Die Analogielehre Meister Eckharts in der Verteidigungsschrift". Dans Meister Eckhart in Erfurt, édition de Andreas Speer, Lidia Wegener. Berlin: De Gruyter, 2005, 356-389.

62 Cf. Burkhard Mojsisch. Meister Eckhart. Analogie, Univozität und Einheit. Hamburg: Felix Meiner, 1983.

63 Eckhart. Prol. gen. n.9, LW 1,1, 154,6-12 (OLME 1, 51).

64 Peut-être évite-t-il de se référer au «sage» pour ne pas induire à l'erreur, car «le sage» est la désignation qu'il emploie pour l'auteur du Livre de la Sagesse, ou celui 
nous sommes nous est donné, mais d'une autre, qu'une fois «justes ou sages», et en tant que tels, nous sommes «un» avec la Justice et la Sagesse, qui revient à Dieu. Il l'exprime ainsi: "celui qui 'est' dans la Sagesse est sage, et celui qui 'demeure' dans la Justice est juste» ${ }^{65}$.

De ce fait, il mentionne que l'âme «en tant qu'elle participe de la nature divine produit ce qui est à partir de ce qui n'est pas» ${ }^{66}$, car son intellect peut penser des choses qui n'existaient pas (In Sap. n.297) ${ }^{67}$. Le Thuringien se réfère ici à l'image de Dieu dans l'être humain, qui est créé capable de Lui (capax dei) —notion augustinienne qu'Eckhart assume. L'image se condense dans le plus élevée de l'âme, qu'il nomme ici intellectus, et ailleurs: le «fond», un «quelque chose dans l'âme», l'«essence de l'âme».

La pensée d'Eckhart s'éclaircit dans son commentaire de Sg 16,25: Ta grâce qui est la nourricière de tous (Omnium nutrici gratiae tuae) (In Sap. n.272-274). Nous avons déjà invoqué qu'il maintient que «tout ce que Dieu opère dans la créature est grâce donnée gracieusement (gratia gratis datur)», et donc le fait d'être créés capables de Lui appartiendrait à cette grâce. Mais il y a aussi la gratia gratum faciens, la «grâce qui rend gracieux, et qui est dite surnaturelle» (In Sap. n.273). Eckhart l'explique avec un exemple pris de la nature: un agent pour pouvoir agir, par exemple pour faire brûler, doit recevoir d'abord en lui-même la forme et l'être du feu. Et il conclut:

«Pareillement dans les actions spirituelles, il est nécessaire que l'âme elle-même reçoive l'être divin, afin qu'elle agisse divinement et spirituellement. Car qui n'est pas juste, n'agit pas justement. Or cet être divin donne la grâce qui rend gracieux à l'essence même de l'âme» ${ }^{68}$.

Eckhart signale que l'âme du juste doit recevoir la justice pour être juste, et ceci suppose une "grâce qui nous rend gracieux». Mais si la sagesse du sage ou la justice du juste ne sont que des dons, qu'en serait-t-il

de L'Ecclésiastique. Cette relation le concret et l'abstrait est au cœur de son enseignement: "Qui comprend la distinction entre la justice et le juste — dit Eckhart- comprend tout ce que je dis». Eckhart. Sermon 6, DW 1, 105.

65 Eckhart. Par. Gen. n.10, LW 1,2, 349,12-13 (JCL, 85).

66 Eckhart. In Sap., Tabula, LW II, 318,16 (JCL, 67).

67 Eckhart semble dire que dans l'acte de penser et créer de nouvelles idées, l'intellect humain participe de l'Intellect divin, et donc toute intellection, chez l'homme, est une action de Dieu en nous.

68 Eckhart. In Sap. n.273, LW 2, 603,1s (JCL, 248). 
la liberté humaine? Le Thuringien dit alors que «l'être divin donne la grâce qui rend gracieux à l'essence même de l'âme», mais elle n'est pas à l'origine de l'action, qui revient à l'homme, car, selon la pensée commune de l'époque, ce n'est pas l'essence mais la personne ou le suppôt qui agit, bien que celui-ci le fait car il en a la capacité. En d'autres mots, Dieu peut nous donner la grâce d'être sages ou justes, mais il nous revient de la mettre en œuvre (In Sap. n.273).

Eckhart poursuit son explication, en précisant que cette grâce tombe dans le seul intellect, «en tant qu'il participe à la nature divine et en a la saveur, et qu'il est l'image ou bien à l'image de Dieu» ${ }^{69}$, c'est-à-dire, la cime de l'âme capable de Dieu. C'est donc là où se trouve la vérité (veritas est in intellectu), mais non en tant qu'intellect naturel, mais «en tant qu'intellect qui participe à l'Intellect divin ${ }^{70}$. Voici un exemple de l'in quantum eckhartien, l'importance que le Thuringien donne aux différentes perspectives. Ce n'est pas la raison naturelle qui saisit et possède la Vérité, qui n'est autre que Dieu, mais l'intellect en tant qu'image de Dieu. Eckhart allègue la Métaphysique d'Aristote (VI, 4, 1027b25), qui signale que le vrai et le faux ne sont pas dans les choses, mais dans l'intellect. Il recourt également à la pensée de Platon et d'Augustin, qui considèrent que «l'intellect, la vérité, la vertu et la science proviennent d'un monde et d'une région au-dessus de la nature», tandis que la perception des sens, le vraisemblable, et l'opinion appartiennent à la région de la nature et au monde inférieur ${ }^{71}$.

\subsection{SAgESSE TERRESTRE - SAgESSE ÉTERNELLE}

Même si Eckhart ne semble pas s'intéresser à préciser les différences entre science et sagesse, il propose aussi deux sortes de sagesse dans ses sermons allemands 95 et 112. Ainsi, le Sermon 95 pour la fête de Sainte Élisabeth de Hongrie, sur le verset: Elle a ouvert sa bouche à la sagesse (Prov. $31,26)$, offre une distinction entre une «sagesse passagère», «terrestre ${ }^{72}$ et

69 Eckhart. In Sap. n.274, LW 2, 604,10s (JCL, 249).

70 Eckhart. In Sap. n.274, LW 2, 604,3. Nous suivons l'interprétation et la traduction de Joseph Koch, dans l'éditon critique, qui met en relation ce texte avec In Eccli. n.10, LW 2, 240,5.

${ }_{71}$ Cf. Augustin. C. acad. III, 17, 37; Retract. I, 3,8.

72 Cf. aussi une allusion aux «savants de la terre» (terrena sapientibus) dans le Sermon latin XLVII n.498, LW 4, 412 (EM, 399). À la suite d'Augustin et du platonisme, 
une «sagesse éternelle» (Sermon 95a); ou bien entre une sagesse qui n'est pas divine, mais qui vient aussi de Dieu, et «une sagesse divine» (95b).

La version A du Sermon 95, qui semble avoir des rajouts par rapport au texte original, s'exprime ainsi par rapport à cette première sorte de sagesse:

«Une sagesse est passagère, elle consiste à se comprendre d'après le temps et à pouvoir s'y adapter, comme un art en quelque sorte, afin que l'on puisse se défendre contre le malheur et que l'on puisse s'adapter à la chance. Celui qui peut agir ainsi devient un homme riche et se nomme bienheureux d'après la sagesse terrestre. Et ainsi la sagesse terrestre est une rosée (tou, une rosée; toum, une odeur, une émanation) de la Sagesse éternelle» ${ }^{73}$.

Cette «sagesse terrestre» est donc une sagesse pratique, un savoir-vivre qui oriente l'action humaine, dont les philosophes grecs avaient parlé. Elle participe aussi en quelque sorte de la Sagesse divine, car elle en est une «rosée» ou une «émanation».

La version B du Sermon 95, qui semble être la version plus proche de l'original, signale que même si cette sagesse n'est pas la Sagesse divine, "pourtant elle est de Dieu comme le rayon [vient] du soleil. C'est un don de Dieu et une «émanation» (toum, ou tou, «rosée») de la nature divine [Sg 7,25b]. Avec cette sagesse, on acquiert en soi de quoi devenir heureux dans cette vie». (STP, 578).

Ainsi, cette «sagesse terrestre» est une sagesse pratique, mais aussi une certaine "émanation» ou une "rosée» de la sagesse divine, en allusion au verset $\operatorname{Sg} 7,25 \mathrm{~b}$.

Le Sermon $112^{74}$ approfondit la question quand le Thuringien explique qu'il y a une sagesse «naturelle» avec laquelle l'on peut s'orienter

aussi présent dans le Livre de la Sagesse, Eckhart donne une connotation négative pour ce qui est terrestre en tant que tel, et qui ce contrapose au divin: «c'est une propriété des choses divines, spirituelles ou célestes que d'être pleines [...], elles sont parfaites et pures formes. [...] Les choses terrestres, en revanche sont matérielles, elles sont passives, imparfaites, nues, misérables et mendiantes du fait même de leur essence». In Gen. n.33, LW 1,2, 92 (OLME 6, 289).

73 Eckhart. Sermon 95a, DW 4,1, 179,11-18 (STP, 572, traduction légèrement modifiée).

74 Eckhart. Sermon 112, DW 4,2, 853,56-64 (notre traduction) «Il existe deux formes de sagesse. La première est naturelle, afin de pouvoir s'orienter envers le temps et les choses muables. C'est pourquoi une personne a le libre arbitre, afin qu'elle puisse 
librement vers le temps et les choses muables ou bien envers Dieu. Et une autre sagesse, celle qui «vient de la présence de Dieu et de la connaissance de Dieu», qui ne peut s'orienter que vers Dieu. Eckhart signale que «la sagesse est une des meilleures choses que Dieu peut donner», car on devient participants, par la sagesse, "de la même béatitude que Dieu est Lui-même et possède en lui-même». Il s'agit donc d'un don qui permet de participer à la vie trinitaire, la constante connaissance de Dieu le Père envers le Fils, et leur amour et jouissance (l'Esprit).

C'est aussi ce que signalent les deux versions du Sermon 95, qui coïncident en ce qui concerne la sagesse qui est divine:

Sermon 95a «L'autre sagesse est éternelle et elle est un jaillissement de la clarté divine et une fontaine de la vérité divine» ${ }^{75}$.

Sermon 95b «L'autre sagesse est un influx de la clarté divine (klârheit), elle est une fontaine de la noblesse divine et elle est Dieu lui-mêmes ${ }^{76}$.

Il y a dans ces expressions une allusion au texte biblique Sg 7, 25b: «Elle (la sagesse) est une émanation pure de la clarté du Dieu tout puissant» (emanatio qucedam est claritatis omnipotentis Dei sincera).

Pour décrire ce qu'est cette sagesse divine, Eckhart met un exemple sympathique et la compare à un bon vendeur, qui met en valeur son produit en vente. La sagesse se vante d'être douce comme le miel (Sir 24,27). Ce que tout le monde souhaite, c'est d'être heureux ou posséder Dieu, —comme l'enseigne Augustin dans son De Trinitate XIII ${ }^{77}$. Et "quoi de plus achevé, parfait et bienheureux que d'être ainsi uni à Dieu?» (In Sap. n.266, 243).

tourner la sagesse vers Dieu ou vers les choses éphémères. La deuxième sagesse vient de la présence de Dieu et de la connaissance de Dieu: elle ne peut se tourner que vers Dieu. La sagesse est l'une des plus grandes choses que Dieu puisse donner. Avec la sagesse, la personne participe de la même béatitude, que Dieu est Lui-même et possède en Lui-même. Voici la béatitude de Dieu: Il se connaît pleinement Lui-même, Il s'aime Lui-même et Il en éprouve du plaisir. C'est avec cette même béatitude que l'homme reçoit le don de Dieu de connaître Dieu, l'aimer et de jouir de son plaisir».

75 Eckhart. Sermon 95a, DW 4,1, 182,54 (STP, 573). (Nous avons modifié légèrement la traduction de Mangin, car klârheit est plus justement clarté que pureté).

76 Eckhart. Sermon 95b, DW 4,1, 184,93-95.

77 Eckhart. In Eccli. n.32, LW 2,261 (B, 37) «Augustin enseigne qu'il n'y a rien que tous veuillent, si ce n'est être heureux ou posséder Dieu. La raison en est qu'en effet la fin de tous est la fin ultime seule, qui est Dieu, la béatitude, le bien suprême». Cf. De Trin. XIII, 3, 6 (édition de Paul Agaësse, Joseph Moingt. Paris: Desclée de Brower [BA 16], 1955, 280-281). 
Il est intéressant de noter que dans ces sermons allemands apparaît la même notion de sagesse présente dans l'œuvre latine (dont nous avons parlé auparavant), comme "perfection spirituelle» qui, à proprement parler appartient à Dieu, et dont les hommes participent (sapientia participata). Car il n'y a pas de différence entre la pensée qu'il exprime dans ses œuvres allemandes ou latines. Mais ces sermons enrichissent la notion de sagesse chez Eckhart, car ils signalent non seulement l'origine de la sagesse (Dieu) mais aussi son objet. Il y a deux sortes de sagesse, l'une «terrestre» ou «naturelle», un savoir-vivre qui gère l'action humaine, et qui peut s'orienter envers les choses ou envers Dieu; et une autre sagesse, don de Dieu, qui vient directement de la présence de Dieu et procure une connaissance de Dieu et le vrai bonheur, la participation à la vie trinitaire et la béatitude éternelle.

\subsection{PHILOSOPHIE - AMOUR DE SA FORME}

Le Maître Thuringien se réfère aussi aux philosophes et s'attache à leur amour de la sagesse pour elle-même. Dans son exposition du verset Sg 8,2: C'est elle que j'ai aimé et cherché à prendre pour épouse, car je sous tombé amoureux de sa forme, il prend l'exemple traditionnel de Pythagore, mais il ne retient pas que celui-ci n'ait pas osé se dire sage et préféra se dire philosophe. Il rappelle qu'étant le plus sage de son époque, «il ne s'est pas glorifié d'être sage, mais d'être philosophe». Le Thuringien vise ici «ceux qui étudient la sagesse, non pour la goûter ni par amour de la sagesse, mais par amour des richesses, des délices et des honneurs» (In Sap. n.201, JCL, 200); ceux-ci ne sont pas «amoureux de sa forme»: à savoir, la vérité. Ainsi Eckhart considère que les vrais philosophes sont ceux qui cherchent à "goûter» (sapere) la Vérité.

Dans Les sermons et leçons sur l'Ecclésiastique, Eckhart revient sur la question et introduit la distinction entre «un sage» et «un philosophe», un ami (amoureux) de la sagesse. Il précise que le but de toute science est la science même, son fruit est connaître et non autre chose, comme l'honneur ou la richesse:

«Toute science qui, dans le savoir (scire) lui-même, ne s'installe pas, ne se repose pas, n'a pas de fruit, ne cherche ni ne trouve, n'est ni libérale ni en vue de soi, mais est mécanique ou adultère, cherchant son fruit en dehors et à côté du savoir (scire). Un tel (savant) n'a pas 
la science et la sagesse comme épouse mais comme concubine, non comme libre, mais comme servante. Il n'est pas l'ami (amator) de sa forme qu'est savoir et être sage [sapere, goûter] [cf. Sap 8,2], mais il est adultère à l'égard de la sagesse [...] C'est pourquoi un tel homme, bien qu'on puisse l'appeler sage, ne peut recevoir ni mériter le nom de philosophe, c'est-à-dire d'ami de la sagesse, mais (il mérite) plutôt celui d'ami des richesses, des honneurs, des commodités, et ainsi de suite, pour lesquels il recherche la sagesse». In Eccli. n.28, LW 2, 250 (B, 32).

Eckhart reprend la confrontation médiévale entre les «sciences théoriques» ou «libérales», qui n'ont pas d'autre intention que la science même, et les «sciences mécaniques» qui ont une orientation pratique. Mais comme dans le texte que nous avons cité précédemment (In Sap. n.201), ce qu'il critique en fin de compte est la poursuite d'un intérêt personnel, de richesses et de succès, au lieu d'un véritable amour pour «la forme de la sagesse» qui est "connaître et goûter» (scire et sapere) la Vérité, "connâ̂tre et goûter» Dieu. Celui-ci est le vrai philosophe.

Ce binôme «connaître et goûter» est évoqué autrement par Eckhart quand il applique à la sagesse l'expression augustinienne d' 'une connaissance accompagnée d'amour» (cum amore notitia) ${ }^{78}$, comme le dit Augustin du Verbe dans son De Trinitate (De Trin. IX, 10, 15, BA 16, 103), adage qu'il attribue explicitement à Augustin à deux occasions ${ }^{79}$.

\subsection{SAPIDA SCIENTIA}

La question de la «saveur» et du «goûter» Dieu, est reprise par Eckhart dans d'autres endroits. Ainsi, dans son Sermon latin sur la fête de Saint Augustin, lors de son premier magistère à Paris, le Dominicain thuringien parle d'une connaissance de Dieu en cette vie «par un miroir et dans la lumière». Il renvoie au De Trinitate I, 2, 4 ainsi qu'au livre IV des Confessions d'Augustin, où celui-ci fait allusion à son extraordinaire expérience de réverbération. Voici l'explication eckhartienne:

78 Eckhart. Sermo LV, n.537, 431,12. Cf. Thomas. Super Sent., lib. 3 d.35 q.2 a.1 qc. 3 arg. 2 «Praeterea in littera dicitur, quod sapientia est in cognitione et dilectione ejus quod semper incommutabiliter manet, quod est Deus».

${ }_{79}$ Cf. Eckhart. In Sap. n.243, 577,4; In Ioh. n.506, LW 3,437,1. 
«Dieu est connu en cette vie par un miroir et dans la lumière, c'est-à-dire quand la lumière divine, par son effet particulier, rayonne sur les puissances cognitives et sur le medium dans la connaissance, élevant l'intellect lui-même vers ce qu'il ne peut naturellement "atteindre". "Car l'esprit de l'homme, du fait de la faiblesse de sa percée, ne s'établit pas dans cette lumière si sublime, excepté s'il n'est d'abord rendu plus pur par la justice de la foi", comme le dit Augustin [dans son] livre Sur la Trinité; et [au livre] VII des Confessions il dit: "Éternelle vérité et vraie charité et chère éternité. C'est toi qui es mon Dieu, après toi je soupire 'jour et nuit' quand pour la première fois je t'ai connue, tu m'as soulevé". Voici de quelle manière il dit qu'il fût élevé, pour lui faire voire qu'il y avait l'être à voir: "moi qui avais vu que j'étais nu ${ }^{80}$; et tu as illuminé (reverberasti) ma faiblesse par la puissance de ton rayonnement sur moi, et j'ai frissonné d'amour et d'effroi et j'ai découvert que j'étais loin de toi dans la région de la dissemblance"». Sermo die b. Augustini n. $5^{81}$.

C'est la lumière divine qui irradie sur les puissances cognitives et élève l'intellect là où il n'arrive pas par soi-même. Cette connaissance a plusieurs effets: l'un d'eux est qu'elle fait «agir de façon méritoire», c'est-à-dire, elle concède par grâce les habitus des vertus, qui conduisent «jusqu'au fruit». Un autre effet est que l'âme reçoit «un avant-goût de la douceur divine», dans «une extase de l'esprit», et ceci «dans le fruit», peut-être une allusion à la béatitude éternelle qui, selon Eckhart, peut déjà se jouir en cette vie.

Le Dominicain thuringien signale alors que «cette connaissance est science ou sagesse, comme une sorte de savoir savoureux (sapida scientia), qui parfois fait entrer l'homme dans une émotion forte» ${ }^{82}$. L'expression sapida scientia renvoie à l'étymologie proposée par Saint Thomas ${ }^{83}$ et commune au Moyen Âge, qui considère l'origine du mot sapientia comme la contraction des deux termes sapi<da> et $<$ sci>entia. Aussi, le fait

${ }^{80}$ Eckhart modifie la version d'Augustin, et au lieu de «et nondum me esse, qui uiderem» il écrit: «et nudum me esse qui videram».

81 Eckhart. Sermo die b. Augustini n.5, LW 5,93,12-94,9. Traduction française de Maurice Mauriège (légèrement modifiée), dans Les mystiques rhénans. Eckhart, Tauler, Suso. Anthologie, dirigé par Marie-Anne Vannier. Paris: Cerf, 2010, 39.

82 Eckhart. Sermo die b. Augustini n.6, LW 595,1 «Haec cognitio scientia vel sapientia, quasi sapida scientia, quae aliquando intromittit hominem in affectum multum».

83 Cf. Thomas d'Aquin, S. Th. I q.43 a.5 resp.2, éd. Léonine, IV, 450 «Et haec proprie dicitur sapientia, quasi sapientia scientia»; $\mathrm{II}^{\mathrm{a}} \mathrm{II}^{\mathrm{ae}}$ q.45 a.2 arg.2, éd. Léonine, VIII, 340 . 
que le verbe latin sapio signifie aussi bien 'savoir' que 'goûter' a facilité la liaison entre la sagesse et saveur ${ }^{84}$.

La fin du texte précédent («...qui parfois fait entrer l'homme dans une émotion forte») évoque l'expérience qu'Augustin partage dans le chapitre $\mathrm{X}$ des Confessions, quand il exclame: "parfois tu me fais entrer dans un sentiment tout à fait extraordinaire au fond de moi, jusqu'à je ne sais quelle douceur qui, si elle devient parfaite en moi, sera je ne sais quoi que cette vie ne sera pas» ${ }^{85}$. Cela éclaircit le sens de ce "savoir savoureux»: c'est une connaissance de Dieu à partir de l'expérience vécue.

Dans le Sermon latin LV sur le verset Sir 14,22: Bienheureux l'homme qui séjournera dans la sagesse, Eckhart éclaire que «la sagesse appartient à l'intellect. Mais [...] elle renferme, presque de façon secondaire, le goût appartenant à l'affectivité» (Sermon LV n.535). Un peu plus loin il renvoie explicitement à Thomas d'Aquin, au livre III de son Commentaire sur les Sentences, (d.35 a.1) et à la deuxième division de la seconde partie de la Somme Théologique ${ }^{86}$, au sujet du don de la sagesse. Cette mention est significative car Eckhart assume, comme Thomas, qu'au-delà de la vertu intellectuelle de la sagesse, qui se met en pratique à travers de l'étude, il y a une sagesse qui est le don de l'Esprit Saint, une certaine connaturalité avec le divin, qui s' «éprouve» (patiens divina), comme celle que Denys l'Aréopagite mentionne par rapport à son maître Hiérothée dans Les noms divins 2, 9.

Dans ce Sermon LV, Eckhart associe plusieurs versets du chapitre 7 du livre de la Sagesse, et y offre diverses possibilités:

«[Bienheureux] celui qui demeurera dans la sagesse (Sir 14,22). Dieu n'aime personne si ce n'est celui qui habite avec la sagesse $(\operatorname{Sg} 7,28)$, à savoir dans le Fils; ou avec la Sagesse, qui est le Fils, il [est celui qui] habite dans le Père; ou avec la sagesse créée, il habite en Dieu, qui est Sagesse incréée. En effet, la sagesse est l'éclat de la lumière (Sg 7,26 a) et une émanation sans tâche de la majesté de Dieu (Sg 7,25b) et l'image

84 Cf. Solignac, 104-106. Cette étymologie apparait déjà chez Grégoire le Grand (Moralia XI, 6, 9) et Isidore de Séville (Etymologiae X, 240). Elle se développe surtout au XII ${ }^{\text {ème }}$ siècle, avec Guillaume de Saint Thierry et Saint Bernard; et elle est aussi partagée par Saint Bonaventure et Saint Thomas.

85 Augustin, Conf. X, 40, 65 (edition de A. Solignac, E. Tréhorel, G. Bouissou. Paris: Desclée de Brower [BA 14], 1962, 258-259).

86 Thomas, $S$. Th. II II q.45 a.2 co. 
de sa bonté $(\mathrm{Sg} 7,26 \mathrm{c})$. Et en raison de cette ressemblance, elle est attirée [à lui] et elle attire l'âme avec elle». Sermon LV n.536, (EM, 432).

Alors, ces versets bibliques peuvent être interprétés de différentes manières. Il peut s'agir de la sagesse divine, qui est le Fils, ou de la sagesse crée, la sagesse humaine participée. La sagesse est "émanation» car elle provient de Dieu, «éclat» car elle le manifeste, et «image» de sa nature. Mais pourquoi est-il dit demeurer dans la sagesse, si elle est dans l'âme? Pour montrer la "pénétration réciproque et l'union la plus grande»-répond Eckhart (n.538). La sagesse, explique-t-il, indique deux choses ensemble: la lumière avec le goût (lumen cum sapore) (n.537) ${ }^{87}$. En raison de la lumière, la sagesse est en nous, soutient Eckhart, mais en raison du goût, nous sommes en elle. Mais pourquoi sommes-nous «dans la sagesse» en raison du goût? Eckhart ne développe pas immédiatement la réponse dans le Sermon latin LV, mais le Sermon allemand 109 peut éclaircir son intention:

«Je vous prie maintenant [...] d'écouter ce que je veux dire et que je n'ai encore jamais dit: Dieu a du goût pour lui-même. Dans le goût par lequel Dieu a un goût intime pour lui-même, il a un goût intime pour toutes les créatures. Avec le goût par lequel Dieu a du goût pour lui-même, il a du goût pour toutes les créatures, non pas en tant que créatures, mais les créatures en tant qu'elles sont divines». Sermon 109 (STP, 452).

Il semble bien que ce goût est l'effet de la suavité de la Sagesse, qui dispose tout avec douceur $(\mathrm{Sg} 8,1)$, dont nous avons parlé précédemment. Le "goût» de Dieu présent dans les créatures, la saveur divine, se retrouve dans leurs raisons (rationes) et leurs formes, qu'elles reçoivent constamment de Lui, dans la création continuée ${ }^{88}$. Celles-ci, de fait, sont dans les choses mais en dehors d'elles, car elles se situent dans le monde intellectuel, dans la pensée divine.

87 Les éditeurs de l'édition critique des Sermons latins d'Eckhart (LW IV, 541 note 7), J. Koch, E. Benz et B. Decker, renvoient à Albert le Grand, Sent. III d.35 a.1, XXVIII 645a «sapientia est quoddam lumen divinorum, sub quo videntur et gustantur divina per experimentum»; et Ibid. a.2, 647a «Sapientia autem stricte dicitur a sapore illius quod simpliciter est sapidum».

88 Cf., par exemple, In Sap. n.283, LW II, 616,3 «L'image, en tant qu'émanation de la forme a, à proprement parler, le goût du bouillonnement — c'est-à-dire, de la vie intradivine-». 
Le binôme lumière / goût présent dans la sagesse, revient aux binômes que nous avons déjà énoncés: notitia cum amore et scire et sapere; la lumière (lumen), la connaissance (notitia) et le connaître (scire) correspondent à la Vérité et au Fils, tandis que sapore, amore, sapere, à l'amour, à l'Esprit. Alors, nous pourrions rencontrer une allusion trinitaire dans l'expression: «dans le goût», dans le Verbe, et «avec le goût», avec l'Esprit.

Le Sermon LV indique plus loin:

«Dieu opère toutes choses avec l'intellect et la sagesse, alors la sagesse le savoure immédiatement et directement, et elle incline vers lui et attire d'autant plus efficacement qu'elle devient une plus pure sagesse». Sermon LV n.539 (EM, 433).

Eckhart renvoie ici à la sagesse divine dans l'âme (sagesse participée). Cette sagesse divine-humaine joue un rôle très important et présente une dynamique: Elle «savoure» Dieu, attire l'âme vers lui et elle «soutient, perfectionne et forme l'âme», affirme le Thuringien dans le Sermon LV. "La sagesse dans l'âme transporte et transforme (transplantat et transformat) l'âme en Dieu, en qui nous nous mouvons, nous vivons et nous sommes (Ac. 17,28), en qui est, bien plus qui est notre béatitude» (n.538, EM, 433). La sagesse a, donc, le rôle d'élévation de l'image de Dieu dans l'âme. Grâce à elle, l'âme devient de plus en plus réceptive à la présence de Dieu en elle, et de plus en plus semblable au Fils (l'Image divine), si détachée de sa forme particulière, et si conforme à celle du Fils, qu'elle reçoive d'être transformée et devienne un avec Lui, et jouisse ainsi de la béatitude. Voici la dialectique de l'image, qui est une autre manière d'exprimer la naissance de Dieu dans l'âme de l'homme noble ou sage, l'homme accompli, qui habite 'dans' et 'avec' la sagesse, et est un avec elle.

\subsection{LA SAGESSE ET LES VERTUS}

Maître Eckhart, tout comme la tradition philosophique et théologique qui lui précède, remarque également l'aspect pratique de la sagesse. Déjà Platon et, après lui, Plotin et Porphyre, s'étaient posée la question de la relation entre les vertus et comment elles se laissent unifier; car elles sont des purifications qui rendent l'être humain semblable à Dieu. De son côté, même si le Thuringien ne propose pas une classification ou 
une systématisation des vertus pour arriver à la béatitude ${ }^{89}$, il reste quand même un Lebemeister, un Maître de vie, et non seulement un Lesemeister, un Maître universitaire. Pour lui, la connaissance 'savoureuse' de Dieu qu'est la sagesse appartient à l'intellect pratique (Sermo die b. Augustini n.6) et doit se mettre en œuvre. Le Maître dominicain reprend explicitement certaines affirmations de Sénèque invitant à la pratique des vertus: «Le plus important critère de la sagesse — dit-il— consiste en (l'accord) des actes avec les paroles ${ }^{90}$. Alors, il est nécessaire de mettre en œuvre «la disposition de la forme» que l'on reçoit gratuitement, comme le témoignait Saint Augustin: «la disposition de la forme chez lui [Augustin] — signale Eckhart, c'est l'accomplissement des vertus dans l'action» (Sermo die b. Aug n.7).

Et il partage avec Augustin la conviction que «la sagesse est en nous couverte et obscurcie par la représentation du sensible» (Sermon LV n.539, EM, 433). C'est pourquoi Eckhart dit dans son Commentaire du Livre de la Sagesse que «la justice, et plus généralement la vertu, est requise pour purifier l'œil de l'âme afin de voir Dieu» (In Sap. n.264, JCL, 242). Il concède une grande valeur à l'humilité: elle accompagne toujours la sagesse (Is 28, 9) (In Sap. n.243), ainsi que les quatre vertus cardinales ${ }^{91}$ et la charité (In Sap. n.243). Ailleurs il cite les sept vertus, cardinales et théologales, qui correspondent aux sept dons de l'Esprit (In Sap. n.205). Dans son Sermon latin pour le jour de Saint Augustin, Eckhart déploie les vertus d'Augustin, et souligne que «la sagesse était constituée pour lui de la vertu» (Sermo die b. Aug n.8). Le Thuringien partage la perspective néoplatonicienne et maintient que même si «les vertus dans leur imperfection sont nombreuses et divisées», "elles sont une dans leur sommet et leur achèvement» (In Sap. n.267, JCL, 244). Il évoque aussi explicitement les quatre grades de vertus de Plotin (Plotinus Platonicus, In Sap. n.263), dont le quatrième grade, celui des vertus exemplaires, «est absolument en Dieu et c'est Dieu lui-même» (Sermon 74) ${ }^{92}$, étant donné qu'il s'agit de

89 Cf. J. Milne. "Meister Eckhart and the Virtues". Medieval Mystical Theology 25/2 (2016): 96-109.

90 Eckhart. In Gen. n.280 (OLME 1, 611).

91 Eckhart. In Sap. n.205 (JCL, 202) «Les quatre vertus cardinales [tempérance, force, prudence et justice] doivent appartenir et être dans chaque homme juste. [...] celui qui possède une vertu les a toutes les quatre. Elles sont en effet connexes»

92 Eckhart. Sermon 74, DW III, 280,9 (TSP, 563) «La vertu a quatre degrés. Le premier fait la percée et écarte du chemin de l'homme toutes choses passagères. Le 
l'idée exemplaire ou la raison de la vertu elle-même. Ainsi, le Thuringien explique qu'il arrive avec les vertus ce qu'il arrive à des vases reliés ou communicants: «si un seul est rempli, tous sont nécessairement remplis pareillement» et il est «impossible d'en remplir un seul sans les remplir tous» (In Sap. n.108, JCL, 144). Par exemple, il soutient que la justice, «en sa perfection, est la sagesse elle-même, ou bien la mère de la sagesse», puisque «la justice en sa perfection est l'Un avec toutes les choses bonnes». (In Sap. n.108, JCL, 144).

En plus des vertus, nous ne pouvons pas oublier l'importance qu'Eckhart concède à l'art du détachement, selon l'exprime Markus Vinzent ${ }^{93}$. Le détachement (abegescheidenheit) et l'abandon (gelassenheit) contribuent à rendre l'âme réceptive à Dieu, car «dans la mesure où tu quittes toutes choses, dans cette même mesure, ni plus ni moins, Dieu pénètre en toi avec tout ce qu'il a» (Entretiens spirituels 4, STP, 728). Le Thuringien explique admirablement la raison et le sens de ce détachement dans son Sermon allemand 102, qui appartient au cycle de sermons sur la naissance de Dieu dans l'âme:

«Notre béatitude ne repose pas sur nos opérations, mais au contraire sur le fait que nous pâtissons Dieu. [...] Oui, d'après un amour sans mesure, Dieu fait reposer notre béatitude dans un pâtir, car nous pâtissons plus que nous agissons, et nous recevons incomparablement plus que nous donnons. Et chaque don prépare l'accueil d'un don nouveau, oui, un don plus fort. Chaque don divin amplifie la réceptivité et le désir de recevoir un [don] plus fort et plus grand». Sermon 102 (STP, 124-125).

Ainsi, les vertus et surtout le détachement permettent la vraie sagesse, c'est-à-dire, la connaissance de Dieu, goûter Dieu: sa naissance dans l'âme.

\subsection{La naissance de Dieu dans L'ÂMe}

En reprenant l'argument de fond sur la notion eckhartienne de la sagesse, si elle vient toujours de Dieu et est toujours en Lui, et est dans les

\footnotetext{
deuxième les soustrait totalement à l'homme. Non seulement le troisième les lui soustrait, il les lui fait totalement oublier, comme si elles n'avaient jamais été, ce qui en est une condition. Le quatrième degré est absolument en Dieu et est Dieu lui-même».

93 Cf. M. Vinzent. The Art of Detachment. Leuven: Peeters, 2011; S. Bara Bancel. Teología mística alemana. Münster: Aschendorff, 2015, 303-316.
} 
hommes uniquement comme une participation de la sagesse divine incréée, la meilleure action humaine consiste à s'efforcer de faire un silence intérieur pour l'accueillir.

C'est ce que va proposer Maître Eckhart, quand il expose le verset du livre de la Sagesse 18,14 (Cum enim quietum silentium contineret omnia, etc.). Il note que «la Sagesse de Dieu, le Verbe», est né, selon la chair, au milieu de la nuit (In Sap. n.279, JCL, 257). Maintenant, par la grâce, il nait aussi dans l'esprit (in mentem) de tout celui qui est en repos et fait silence $^{94}$.

Le Thuringien s'appuie sur trois textes des Confessions de Saint Augustin (Conf. IX 10, 25; IV, 11, 16 et IX, 11, 16), qui invitent l'âme précisément à faire repos et silence. Voici les paroles de Maître Eckhart:

«De plus, la Sagesse vient dans l'esprit quand l'âme se repose du tumulte des passions et de l'occupation causée par les choses de ce monde, quand toutes ces choses-là font silence, et que celle-ci fait silence à toutes [Conf. IX 10,25]. C'est ce que dit Augustin au livre IV des Confessions: "Ne sois pas vaine, mon âme! N'assourdis pas l'oreille de ton cœur par le tumulte de ta vanité. Entends la Parole elle-même (...): ici est l'endroit d'un repos sans trouble (...) Ici, installe ta demeure" [Conf. IV, 11, 16]. Puis, au livre IX [Conf. IX, 11, 16], il dit: "Quoi de semblable à ton Verbe, à notre Seigneur: en Lui toutes choses se maintiennent et se renouvellent?" Et ce qui suit: "Si pour une âme, le tumulte de la chair faisait silence, si les délires de la terre faisaient silence (...) et ceux du ciel, que cette âme soit silencieuse à elle-même, et qu'elle se dépasse sans penser à elle, si se taisaient les songes et les révélations imaginaires (...) et que Lui seul parle (...) de lui-même afin que nous entendions sa Parole"». In Sap. n.280, (JCL, 257-258).

Eckhart retient d'Augustin le repos du «tumulte de la vanité» et «de la chair», des "délires de la terre» et ceux du ciel, des songes, des imaginations, pour pouvoir «entendre la Parole» et y «installer sa demeure». Et plus loin le Thuringien recourt à Avicenne (Métaphisyque VIII, chap. 5) et aussi à Augustin pour expliquer qu'il est nécessaire que tout reste en silence, et que les choses créés et distinctes en tant que telles fassent silence dans l'âme pour qu'elle puisse recevoir Dieu, lui qui est indistinct, et devenir 'un' avec lui (Eckhart, In Sap. n.282, LW 2, 614).

94 Eckhart. In Sap. n.281, LW 2, 612,6 (JCL, 258) «oportet quod quies et 'silentium contineat omnia' ad hoc, ut deus verbum in mentem veniat per gratiam et filius nascatur in anima». 
«Comme le dit Augustin dans le De Trinitate VIII [3, 4] — signale Eckhart-l'âme est naturellement portée vers le bien ce qui est purement et absolument bon. Mais rien de ce qui est ceci ou cela est purement et absolument bon ${ }^{95}$. C'est ce que dit le même Augustin dans le premier livre des Confessions [I, 1, 1], en parlant à Dieu: "Tu nous as fait pour Toi, et notre cœur est sans repos, jusqu'à ce qu'il repose en Toi” ». Eckhart, In Sap. n.282 (LW 2, 615).

Nous pouvons constater qu'Eckhart s'appuie longuement sur Augustin pour élaborer sa pensée, mais il va au-delà de lui et ne parle plus seulement de l'écoute de la Parole, mais d'une naissance de la Parole dans l'âme: le Père, en prononçant son Verbe, l'engendre en elle. En ce faire, il s'appuie explicitement sur Origène ${ }^{96}$. Dans son Sermon allemand 101, qui s'occupe du même verset Sg 18,14 (qui se proclamait dans la liturgie de Noël), Eckhart reprend son propos et le formule avec plus d'élan ${ }^{97}$.

Ainsi, le Thuringien ne suit plus Augustin quand celui-ci regrette, dans les Révisions, d'avoir écrit que l'homme est capable d'atteindre la vie bienheureuse et la connaissance parfaite de Dieu déjà dans la vie présente (Retract. I, 2). Eckhart convient que cette connaissance, cette sagesse, qui est une participation à la vie trinitaire et une naissance, n'a pas lieu en ce monde. Mais, en s'appuyant aussi sur Augustin il dit qu'alors nous ne sommes plus dans ce monde ; et il le fait en reliant deux citations augustiniennes, De moribus Ecclesiae et le De Trinitate (De Trin. IV, 20, 27-28, et De mor. eccl. 11, 19), qu'il cite trois fois complètement, et même reprend l'argument lors de son Procès à Cologne ${ }^{98}$ :

95 Cf. Augustin, De Trin. VIII, 3, 4 (BA 16, 33). «Ceci est bien, cela est bien. Supprime-le ceci et le cela (hoc et illud), et vois, si tu peux, le bien même: alors tu verras Dieu, qui ne tient pas sa bonté d'un autre bien, mais est la bonté de tout bien».

96 Cf. Elisabeth Boncour. Maître Eckhart, lecteur d'Origène. Sources, exégèse, anthropologie, théogénésie. Paris: Vrin, 2019.

97 Eckhart. Sermon 101 (TSP, 95) «Saint Augustin dit: Que cette naissance se produise toujours mais qu'elle ne se produise pas en moi, en quoi cela peut-il bien m'aider? En revanche, qu'elle se produise en moi, cela a beaucoup d'importance». Même si ici Eckhart l'attribue à Augustin, il est en train de citer Origène. Cf. Origène. Homélies sur la Génèse III, 6 (édition de Henri de Lubac, Louis Doutreleau. Paris: Cerf [SC 7bis], 1976, 141). «Car à quoi sert de dire que Jésus n'est venu que dans la chair qu'il a prise de Marie et de ne pas montrer aussi qu'il est venu dans ma chair à moi?».

98 Eckhart. In Ioh. n.286, LW 3, 240,2; In Ioh. n.450, LW 3, 385,4; [3] In Ioh. n.469, 402,2; Proc. Col. II n.135, LW 5, 350,3. 
"Augustin dit dans son De moribus ecclesiae [11, 19] "l'esprit, en s'adhérant à Dieu, est bien au-dessus du monde entier". Et dans le De Trinitate IV, 20, [28], Augustin dit de même: Le Verbe c'est fait chair. "Lorsque au cours du temps quelqu'un le perçoit" “on dit en effet qu'il est envoyé [du Verbe], mais pas en ce monde", "d'ailleurs nous-mêmes dans la mesure, à nous possible, où nous saisissons quelque chose de l'éternel, nous ne sommes pas en ce monde; les esprits de tous les justes, même ceux qui vivent encore dans ce corps, pour autant qu'ils éprouvent le divin (in quantum divina sapiunt), ne sont pas dans ce monde"». Eckhart, In Ioh. n.286, LW 3, 240,1 (notre trad.)

Finalement, «quand l'âme parvient à la connaissance que Dieu est dissemblable à toute créature —explique Eckhart dans le Sermon 95b—, elle [y] parvient dans une merveille, [...] dans un silence. Avec douceur, Dieu s'abaisse dans l'âme, et avec la grâce il se répand sur elle» (STP, 582). Mais pour cela l'âme doit «mourir dans la souffrance, comme notre Seigneur», "cette mort de l'âme concerne, dit-il, à la connaissance de Dieu» (Sermon 95b, STP, 582), une connaissance qui en fait est au-delà des facultés humaines, c'est une «non-connaissance», une ignorance (Sermon 101) ${ }^{99}$. Car l'âme «saisit Dieu dans sa substance pure et dépouillé», et alors «l'homme a une seule science avec la science de Dieu et une seule opération avec l'opération de Dieu et une seule connaissance avec la connaissance de Dieu» (Sermon 40, STP, 449). Il s'agit là de la connaissance de la personne accomplie: "Connaître Dieu et 'le savoir', c'est la justice achevée» (In Sap. n.262-263). «En effet — dit Eckhart— quoi de plus achevé, parfait et bienheureux que d'être ainsi uni à Dieu?». (In Sap. n.266, 243). Mais il s'agit d'une connaissance et une sagesse paradoxale car, dans cette union, les facultés humaines sont en silence, et la personne n'en est pas consciente, et quand elle revient à ses facultés, elle n'en est plus à ce stade d'union à Dieu (cf. De l'homme noble).

99 Cf. F. Löser. "Vom Nichtwissen Meister Eckharts. Eine Skizze". Dans Geschichte(n) des Wissens. Festschrift für Wolfgang E. J. Weber zum 65. Geburtstag, édition de M. Häberlein, S. Paulus, G. Weber. Augsburg: Wissner Verlag, 2015, 41-58. 


\section{CONCLUSION}

Au long de notre analyse des textes où Eckhart aborde la question de la sagesse nous avons constaté que le Maître thuringien s'appuie aussi bien sur la conception de sagesse qui apparaît dans l'Écriture, spécialement dans les livres sapientiaux (la sagesse divine personnifiée), que sur les réflexions des philosophes (Platon, Plotin, le Liber de causis, Aristote) et sur la tradition théologique antérieure à lui. Alors, il reprend et approfondit certains propos d'Augustin et d'Origène, ainsi que de Thomas d'Aquin mais, en ce faisant, il offre une pensée originale,

En premier lieu, la Sagesse chez Eckhart désigne Dieu (sapientia increata), la nature divine, un attribut divin et aussi le Fils, «sagesse de Dieu» (1Cor 1,24). La Sagesse est «une émanation de Dieu», «une image de sa bonté» et «l'éclat de sa lumière» (Sg 7,25-26). Le Thuringien semble ne pas apprécier les délimitations et les divisions, et ses affirmations sur la Sagesse divine peuvent fréquemment s'entendre aussi bien du Fils que de l'essence divine pure. En tant que Sagesse, Dieu est pureté et indistinction, il est «totalement dedans» et «totalement en dehors de toute chose» (In Sap. n.135), et il «dispose avec douceur toutes les chose» (Sg 8,1). La Sagesse divine est, pour Eckhart, le regard de Dieu sur les choses, qui leur confère leur essence. Il s'agit de la «maternité» de la Sagesse divine, qui «dispose avec douceur toutes les choses», c'est-à-dire, de sa création continuée, qui leur donne l'être et maintient et conserve la création. La création a lieu «dans le Principe», dans le Verbe-Sagesse, où se retrouvent les raisons idéelles de toutes les choses, leur "être virtuel», c'est-à-dire, l'être qu'elles ont dans leur cause, à différence de leur «être formel», l'être naturel au sein de la matière.

En deuxième lieu, la sagesse chez les hommes vient toujours de Dieu (sapientia participata): «Toute âme devient sage par sa participation à la Sagesse» (Prol. Gen. n.9). Car la sagesse est incréée et incréable (Qu. Par. I n.4) et a une dimension transcendantale. Eckhart n'insiste pas sur la différence entre sagesse et science, mais il introduit une distinction entre la «sagesse terrestre» ou «naturelle», un savoir-vivre qui oriente l'action humaine, et «une sagesse éternelle» ou «divine» (Sermon 95); bien que la première est aussi «une rosée» de la Sagesse de Dieu, mais elle peut s'orienter envers Lui ou envers les choses. L' «autre sagesse» vient directement de la présence de Dieu, il s'agit alors du don divin de la sagesse, «une sorte de savoir savoureux» (Sermo die b. Agustini), «l'avant-goût de 
la douceur divine», qui n'est autre que la connaissance de Dieu par expérience, l'union immédiate à Dieu, autrement dit, la naissance de Dieu au fond de l'âme. Mais pour arriver à cette sagesse, les vertus conduisent à purifier l'âme. L'humilité, la charité, la justice, etc., toutes les vertus se relient et ne «sont qu'une dans leur sommet et leur achèvement» (In Sap. n.267). Elles rendent l'âme ressemblante à Dieu pour qu'elle puisse «goûter la sagesse divine» (Sermon 95b); c'est-à-dire, pour que Dieu puisse naître en elle (Sermon 101, Sermon 40, In Sap n.280), quand toutes les choses font silence. Cette sagesse, cette connaissance de Dieu est, en fait, une ignorance, une non-connaissance, qui a lieu au-delà des facultés humaines, au plus pur, au fond de l'âme (Sermon 101). Alors, la personne jouit déjà de la béatitude éternelle, car elle est «dans le maintenant présent de l'éternité» (Sermon 24). Eckhart s'appuie sur Augustin (De Trin. IV, 20, 27-28, et De mor. eccl. 11,19) pour aller au-delà de lui et dire qu'alors la personne n'est plus dans ce monde, en tant qu'elle éprouve et goûte le divin (in quantum divina sapiunt).

En somme, le Thuringien conçoit la sagesse humaine comme un don de Dieu: une participation à la Sagesse divine et à la vie trinitaire, un «savoir» et "goûter» Dieu, une naissance. Le sage, en tant que tel, est né et fils de la Sagesse: le Père l'engendre en lui, au plus profond et pur de son âme, et ceci accompagné de la jouissance de l'amour, de l'Esprit.

\section{RÉFÉRENCES}

Aersten, Jan A. "La doctrine des transcendantaux de Maître Eckhart". En Maître Eckhart, dirigido por Julie Casteigt, 21-39. Paris: Cerf, 2012. Albertus Magnus. Super Dionisii mysticam theologiam et epistulas, editado por Paul Simon. Monasterii Westfalorum [Münster]: Aschendorff, 1978. Augustin d'Hippone. Les Confessions, editado por A. Solignac, E. Tréhorel, G. Bouissou. 2 Vol. Paris: Desclée de Brower, 1962.

Augustin d'Hippone. La Trinité. Le mystère. Livres I-VII, editado por E. Hendrikx, M. Mellet, Th. Camelot. Paris: Desclée de Brower, 1955. Augustin d'Hippone. La Trinité. Livres VIII-XV, editado por P. Agaësse, J. Moingt. Paris: Desclée de Brower, 1955.

Bara Bancel, Silvia. Teología mística alemana. Münster: Aschendorff, 2015. 
Boncour, Élisabeth. "La source origénienne de la naissance du Verbe en l'âme et le traitement eckhartien". En Maître Eckhart, lecteur des Pères grecs, dirigido por Marie-Anne Vannier, 43-57. Paris: Beauchesne, 2020.

Boncour, Élisabeth. Maître Eckhart, lecteur d'Origène. Sources, exégèse, anthropologie, théogénésie. Paris: Vrin, 2019.

Brunner, Fernand. "Lanalogie chez Maître Eckhart". Freiburger Zeitschrift für Philosophie und Theologie 16 (1969): 333-349.

Chevalier, Philippe, ed. Dionysiaca: recueil donnant l'ensemble des traductions latines des ouvrages attribués au Denys de l'Aréopage. Paris: Desclée de Brower, 1937.

Damascenus, Johannes. De fide orthodoxa. Burgundionis Versio, editado por Eligius M. Buytaert. New York: Franciscan Institute Publications, 1955.

Duclow, Donald F. "Meister Eckhart on the Book of Wisdom: Commentary and Sermons". Traditio 43 (1987): 215-235. https://doi.org/10.1017/ S036215290001254X

Eckhart, Meister. Die deutschen und die lateinischen Werke. Die deutschen Werke, editado por Josef Quint, y Georg Steer. 5 vols. Stuttgart: Kohlhammer, 1936-2003.

Eckhart, Meister. Die deutschen und die lateinischen Werke. Die lateinischen Werke, editado por Ernst Benz, Karl Christ, Bruno Decker, Bernhard Geyer, Joseph Koch, Heribert Fischer, Erich Seeberg, Loris Sturlese y Albert Zimmermann. 5 vols. Stuttgart: Kohlhammer, 1936-2015.

Eckhart, Maître. Commentaire du Livre de la Sagesse, traducido por Jean Claude Lagarrigue, Jean Devriendt. Paris: Les Belles Lettres, 2015.

Eckhart, Maître. La mesure de l'amour. Sermons parisiens, traducido por Eric Mangin. Paris: Seuil, 2009.

Eckhart, Maître. L'ouvre latine de Maître Eckhart. Vol. 1, Commentaire de la Genèse, précédé des Prologues, traducido por Fernand Brunner, Alain de Libéra, Éduard Wéber, y Émilie Zum Brun. Paris: Cerf, 1984.

Eckhart, Maître. L'ouvre latine de Maître Eckhart. Vol. 6, Commentaire de l'évangile selon Jean. Le prologue, traducido por Alain de Libéra, Éduard Wéber, y Émilie Zum Brun. Paris: Cerf, 2007.

Eckhart, Maître. Sermons et leçons sur l'Ecclésiastique, traducido por Fernand Brunner. Genève: Ad Solem, 2002.

Eckhart, Maître. Sermons, traités, poème, traducido por Jeanne Ancelet-Hustache, y Eric Mangin. Paris: Seuil, 2015.

La Bonnardière, Anne-Marie. Biblia Augustiniana. AT. Le Livre de la Sagesse. Paris: Études Augustiniennes, 1970. 
Löser, Freimut. "Vom Nichtwissen Meister Eckharts. Eine Skizze". En Geschichte(n) des Wissens. Festschrift für Wolfgang E. J. Weber zum 65. Geburtstag, editado por Marc Häberlein, Stephan Paulus, y Gregor Weber, 41-58. Augsburg: Wissner Verlag, 2015.

Milne, Joseph. "Meister Eckhart and the Virtues". Medieval Mystical Theology 25, n. ${ }^{\circ} 2$ (2016): 96-109. https://doi.org/10.1080/20465726.2 016.1253959

Mojsisch, Burkhard. Meister Eckhart. Analogie, Univozität und Einheit. Hamburg: Felix Meiner, 1983. https://doi.org/10.28937/978-3-7873-2750-8

Origène. Traité des principes. Livres I et II, traducido por H. Crouzel et M. Simonetti. Paris: Éditions du Cerf, 1978.

Origène, Homélies sur la Genèse, editado por Henri de Lubac, Louis Doutreleau. Paris: Cerf, 1976.

Pattin, Adriaan, ed. Le 'Liber de causis'. Édition établie à l'aide de 90 manuscrits avec introduction et notes. Leuven, 1967.

Schiffhauer, Angela. "nos filii dei sumus analogice. Die Analogielehre Meister Eckharts in der Verteidigungsschrift". En Meister Eckhart in Erfurt, editado por A. Speer, y L. Wegener, 356-389. Berlin: De Gruyter, 2005.

Solignac, Aimé. "Sagesse antique, sagesse chrétienne". En Dictionnaire de Spiritualité. Vol. 14, 96-114. Paris: Beauchesne, 1990.

Speer, Andreas. "Philosophie als Lebensform? Zum Verhältnis von Philosophie und Weisheit im Mittelalter". Tijdschrift voor Filosofie 62 (2000): 3-25.

Speer, Andreas. "Sapientia nostra. Zum Verhältnis von philosophischer und theologischer Weisheit in den Pariser Debatten am Ende des 13. Jahrhunderts". En Nach der Verurteilung von 1277. Philosophie und Theologie an der Universität von Paris im letzten Viertel des 13. Jahrhunderts, editado por Jan A. Aertsen, Kent Emery, y Andreas Speer, 248-275. Berlin: De Gruyter, 2001.

Speer, Andreas. "Weisheit bei Augustinus und Meister Eckhart". En Meister Eckhart und Augustinus, editado por Rudolf K. Weigand, y Regina D. Schiewer, 1-15. Stuttgart: Kohlhammer, 2011.

Sturlese, Loris. "Acta et regesta vitam mag. Echardi illustrantia”. En Meister Eckhart. Die lateinischen Werke. Vol. 5, editado por Albert Zimmerman, y Loris Sturlese, 153-194. Stuttgart: Kohlhammer, 2006.

Sturlese, Loris, ed. Studie sulle fonti di Meister Eckhart. 2 vols. Fribourg: Dokimion 34 y 37, 2008 y 2013.

Vannier, Marie-Anne. Maître Eckhart prédicateur. Paris: Beauchesne, 2018. 
144 SILVIA BARA BANCEL, «SAGESSE INCRÉÉE» ET «SAGESSE PARTICIPÉE»...

Vannier, Marie-Anne, ed. Les mystiques rhénans. Eckhart, Tauler, Suso. Anthologie. Paris: Cerf, 2010.

Vinzent, Markus. The Art of Detachment. Leuven: Peeters, 2011.

Wackernagel, Wolfgang. Poésies mystiques et prière de Maître Eckhart. Genève: Ad Solem, 1998.

Wéber, Édouard-Henri. "La théologie de la grâce chez Maître Eckhart". Revue des sciences religieuses 70, n. 1 (1996): 48-72. https://doi.org/10.3406/ rscir.1996.3346 